TRANSACTIONS OF THE

AMERICAN MATHEMATICAL SOCIETY

Volume 354, Number 10, Pages 3973-4013

S 0002-9947(02)03030-

Article electronically published on June 10, 2002

\title{
EMBEDDINGS UP TO HOMOTOPY OF TWO-CONES IN EUCLIDEAN SPACE
}

\author{
PASCAL LAMBRECHTS, DON STANLEY, AND LUCILE VANDEMBROUCQ
}

\begin{abstract}
We say that a finite CW-complex $X$ embeds up to homotopy in a sphere $S^{n+1}$ if there exists a subpolyhedron $K \subset S^{n+1}$ having the homotopy type of $X$. The main result of this paper is a sufficient condition for the existence of such a homotopy embedding in a given codimension when $X$ is a simply-connected two-cone (a two-cone is the homotopy cofibre of a map between two suspensions).

We give different applications of this result: we prove that if $X$ is a two-cone then there are no rational obstructions to embeddings up to homotopy in codimension 3. We give also a description of the homotopy type of the boundary of a regular neighborhood of the embedding of a two-cone in a sphere. This enables us to construct a closed manifold $M$ whose Lusternik-Schnirelmann category and cone-length are not affected by removing one point of $M$.
\end{abstract}

\section{INTRODUCTION}

We are interested in embeddings "up to homotopy" of a finite CW-complex in a sphere:

Definition 1. We say that a finite CW-complex $X$ embeds up to homotopy (or $h$-embeds) in a sphere $S^{n+1}$ if there exists a compact subpolyhedron $K \subset S^{n+1}$ of the same homotopy type as $X$. We write then $X \stackrel{h}{\subset} S^{n+1}$ or $X \simeq K \subset S^{n+1}$.

Embeddings up to homotopy differ drastically from inclusions of subpolyhedra or PL-embeddings in a sphere (PL is for piecewise-linear) . For instance Cooke [1] gives examples for arbitrarily large $i$ of two-cell complexes $X$ such that $X \not \subset S^{n+1}$ but $\Sigma^{i} X \stackrel{h}{\subset} S^{n+1}$. On the other hand, it is well known that if $X$ is a polyhedron such that $\Sigma^{i} X$ PL-embeds in $S^{n+1}$, then $X$ PL-embeds in $S^{n-i+1}$. In the beginning of Section [7 of the present paper we also construct examples for arbitrarily large $i$ of two-cell complexes $X$ such that $\Sigma X \stackrel{h}{\subset} S^{n+1}$ but $X \not \subset{ }^{h} S^{n+i+1}$.

A natural question is

Problem A: Given a finite CW-complex $X$, what is the smallest dimension $n$ such that $X$ h-embeds in a sphere $S^{n+1}$ ?

Received by the editors February 22, 2000 and, in revised form, June 1, 2001.

2000 Mathematics Subject Classification. Primary 57R40, 55P25, 55Q25, 55M30.

Key words and phrases. Two-cone, embedding, cone-length, homotopical boundary.

P.L. is chercheur qualifié au F.N.R.S.

D.S. was supported by CNRS at UMR 8524 "AGAT", Université de Lille 1.

L.V. was supported by a Lavoisier fellowship and an Alexander von Humboldt fellowship. 
This is an old problem. It was initiated by Thom (39 and Chapter 3 of 35]) and pursued by many authors, for example in [32], [27], [28], [17], [36], [11], [10], [16], 22]. In his important paper [11] Cooke gives a necessary and sufficient condition for the existence of an h-embedding of a two-cell complex in a sphere:

Theorem 2 (Cooke). Let $p \geq 2, q \geq p+2$ and $n \geq q+2$. The two-cell complex $S^{p} \cup_{f} e^{q} h$-embeds in $S^{n+1}$ if and only if there exist $\beta \in \pi_{n-p-1}\left(S^{n-q}\right)$, $\phi \in \pi_{q-1}\left(S^{p} \vee S^{n-q}\right)$ and $\psi \in \pi_{n-p-1}\left(S^{p} \vee S^{n-q}\right)$ such that

$$
\left[S^{p} \circ f+\phi, S^{n-q}\right]=\left[S^{n-q} \circ \beta+\psi, S^{p}\right]
$$

where we denote by $S^{p}$ and $S^{n-q}$ the inclusions of each sphere into the wedge $S^{p} \vee S^{n-q}$

Connolly and Williams ([10], Section 5) state the following more explicit criterion for h-embedding of two-cell complexes in a sphere, valid only for large enough codimensions:

Theorem 3 (Connolly-Williams). Let $q \geq p \geq 2, n \geq q+2$ and $f \in \pi_{q-1}\left(S^{p}\right)$. Suppose that $n \geq 2 q-2 p-2$. Then $S^{p} \cup_{f} e^{q} h$-embeds in $S^{n+1}$ if and only if $\Sigma^{n-q} f \in \pi_{n-1}\left(S^{n+p-q}\right)$ desuspends $p$ times.

In the present paper we study h-embeddings of finite two-cones:

Definition 4. A two-cone is the homotopy cofibre of a map $f: \Sigma A \rightarrow \Sigma B$ between two suspensions. This two-cone is called finite if $A$ and $B$ are finite $\mathrm{CW}$-complexes.

Our main result (Theorem 231) is a sufficient condition for the existence of an h-embedding of a given simply-connected finite two-cone $X=\Sigma B \cup_{f} C \Sigma A$ in a sphere. Let $f^{\natural}: A \rightarrow \Omega \Sigma B$ be the adjoint of $f: \Sigma A \rightarrow \Sigma B$. Our sufficient condition is expressed in terms of desuspendability of the image of $\Sigma f^{\natural}$ by a certain SpanierWhitehead duality isomorphism. In the case of a two-cell complex, that is for $f: S^{q-1} \rightarrow S^{p}$, we can also express this condition in terms of desuspendability of the James-Hopf invariants. Recall indeed that, via the James equivalence $\Sigma \Omega S^{p} \simeq$ $\bigvee_{k=1}^{\infty} S^{k(p-1)+1}$, we have $\Sigma^{n-q} f^{\natural}=\sum_{k=1}^{\infty} \Sigma^{n-q-1} \gamma^{k}(f)$, where $\gamma^{k}(f): S^{q-1} \rightarrow$ $S^{k(p-1)+1}$ are the James-Hopf invariants of $f$. We will prove (see Corollary 24):

Corollary 5. Let $q \geq p \geq 2, n \geq q+2$, and $f \in \pi_{q-1}\left(S^{p}\right)$. If $\sum^{n-q} f^{\natural} \in$ $\pi_{n-2}\left(\bigvee_{k=1}^{\infty} S^{k(p-1)+n-q}\right)$ desuspends $p$ times, or equivalently, if $\Sigma^{n-q-1} \gamma^{k}(f)$ desuspends $p$ times for any $k \geq 1$, then $S^{p} \cup_{f} e^{q} h$-embeds in $S^{n+1}$.

In contrast with the result of Connolly-Williams, the only restriction on the codimension in our corollary is that it must be greater than 3 , which is a hypothesis common to all results on h-embeddings. Corollary 5 permits us for instance to exhibit an h-embedding of a space $S^{8} \cup_{f} e^{30}$ in codimension 3 (see Example 25) whose existence does not follow from the theorem of Connolly and Williams stated above. Corollary 5 also gives a larger range of codimensions than in Theorem 3 (as soon as $p \geq 5$ ) in which the desuspendability of a certain suspension of $f$ guarantees the existence of an h-embedding (see Corollary 26):

Corollary 6. Let $q \geq p \geq 2, n \geq q+2$, and $f \in \pi_{q-1}\left(S^{p}\right)$. Suppose also that $n \geq 2 q-3 p+3$. Then $S^{p} \cup_{f} e^{q} \stackrel{h}{\complement} S^{n+1}$ if $\Sigma^{n-q-1} f$ desuspends $p$ times.

Notice that we lose one dimension in comparison with the result of Connolly and Williams. This shift of dimension comes from the fact that the h-embedding that we get is "tractable" (see Definition 12 below). 
There are well-known obstructions to the existence of an h-embedding of a finite CW-complex in a sphere $S^{n+1}$. For example Thom ([39]; 35], Chapter 3) described such obstructions in terms of Steenrod operations, Peterson and Stein ([28]) constructed other obstructions using secondary cohomology operations, and there is the first obstruction of Habegger, which is the same as those of Thom ([16], Theorem III). As far as we know all such obstructions are "torsion". We prove actually that there are no rational obstructions for the existence of an h-embedding of a two-cone in a sphere of codimension 3. More precisely:

Theorem 7. Let $X$ be a finite simply-connected two-cone of dimension $n$. Then there exists a finite $C W$-complex $X^{\prime}$ of the same rational homotopy type as $X$ and which h-embeds in $S^{n+3}$.

We conjecture that this theorem is still true without the hypothesis on the conelength of $X$. Observe that this phenomenon holds only for embeddings up to homotopy: if $M$ is a closed PL-manifold then the Pontrjagin classes do provide rational obstructions for piecewise-linear embeddings.

Another notion related to h-embedding in a sphere is that of Euclidean thickening (which is a special case of the notion of thickening introduced by Wall in 40]):

Definition 8. Let $X$ be a simply-connected finite CW-complex. A Euclidean thickening of $X$ of dimension $n+1$ is a compact PL-submanifold $N$ of codimension 0 of $S^{n+1}$ which has the same homotopy type as $X$ and is such that $\pi_{1}(\partial N)=0$.

Of course, if $X$ admits a Euclidean thickening of dimension $n+1$, then $X \stackrel{h}{\subset} S^{n+1}$. Conversely, if $X \simeq K \subset S^{n+1}$ is an h-embedding, then any regular neighborhood (see [31] for a definition) $N$ of $K$ is a Euclidean thickening of dimension $n+1$ of $X$. This regular neighborhood is called the thickening induced by the h-embedding $X \simeq K \subset S^{n+1}$.

Definition 9. A Euclidean homotopy boundary of dimension $n$ of $X$ is the boundary $\partial N$ of a Euclidean thickening $N$ of $X$ of dimension $n+1$.

Thus a Euclidean homotopy boundary is a closed manifold which is an invariant of the h-embedding in the sense that it depends only on the choice of the polyhedron $K$ homotopy equivalent to $X$ and on the isotopy class of the embedding $K \subset S^{n+1}$. This suggests the following:

Problem B: Given a finite CW-complex $X$, which are the homotopy types of Euclidean homotopy boundaries of $X$ ?

This problem was already raised by Klein [22], who solved it when $X$ is a suspension. In the present paper we give a description of some Euclidean homotopy boundaries of two-cones (Corollary [30). In the stable range, that is when $n \geq 2 \operatorname{dim}(X)+1$, the Euclidean homotopy boundary is essentially unique because of the following:

Theorem-Definition 10 (Wall, 40]). If $X$ is a simply-connected finite $C W$-complex of dimension $m$ and if $n \geq \max (5,2 m+1)$, then there is a unique (up to $P L$ homeomorphism) Euclidean thickening $N$ of $X$ of dimension $n+1$. This thickening $i s$ called the trivial thickening of $X$ of dimension $n+1$.

This uniqueness result justifies the following: 
Definition 11. If $X$ is a simply-connected finite CW-complex of dimension $m$, and if $n \geq \max (5,2 m+1)$ the boundary of its trivial thickening of dimension $n+1$ is called the trivial homotopy boundary of $X$ of dimension $n$ and is denoted by $\mathcal{B}^{n}(X)$.

Thus in the stable range Problem B becomes: when $n \geq 2 \operatorname{dim} X+1$, describe the homotopy type of $\mathcal{B}^{n}(X)$ from that of $X$. We give a complete solution to this problem when $X$ is a simply connected finite two-cone (Corollary 32).

With the above notion of Euclidean thickening we can recall the following definition

Definition $12([12])$. A Euclidean thickening $N$ is tractable if the inclusion $i$ : $\partial N \hookrightarrow N$ admits a homotopy section $\sigma: N \rightarrow \partial N$. That is, there exists a map $\sigma: N \rightarrow \partial N$ such that $i \circ \sigma \simeq \mathrm{id}_{N}$. An h-embedding is tractable if its induced thickening is.

It is clear ([12], Proposition 2.2) that if $A \simeq K \subset S^{n+1}$ is an h-embedding, then by composing with the inclusion of the equator $S^{n+1} \times\{0\} \subset S^{n+2}$ we get a tractable h-embedding $A \simeq K \times\{0\} \subset S^{n+2}$; this h-embedding $A \stackrel{h}{\subset} S^{n+2}$ is called the suspension of $A \stackrel{h}{\subset} S^{n+1}$. In all the results of this paper the h-embeddings that we will construct will be tractable.

A last application of our results is devoted to the cone-length and LusternikSchnirelmann category of a closed manifold. Recall that the cone-length of a space $X, \mathrm{Cl}(X)$, is defined in [13] as follows: $\mathrm{Cl}(X)=0$ if and only if $X$ is contractible, and $\mathrm{Cl}(X) \leq n+1$ if and only if there exists a homotopy cofibration $\Sigma^{n} U \rightarrow Y \rightarrow X$ with $\mathrm{Cl}(Y) \leq n$. For example, two-cones are exactly spaces of cone-length less than or equal to 2. The Lusternik-Schnirelmann category of a space $X$, cat $X$, is the smallest integer $n$ such that $X$ can be covered by $n+1$ open sets contractible in $X$. We have the inequalities cat $(X) \leq \mathrm{Cl}(X) \leq \operatorname{cat}(X)+1$, and both equalities can occur (13, 34]). If $M$ is a closed manifold and if $p \in M$, then cat $(M) \leq$ cat $(M \backslash\{p\})+1$ and $\mathrm{Cl}(M) \leq \mathrm{Cl}(M \backslash\{p\})+1$, and moreover, equalities occur for all obvious examples. In the other direction, Rivadeneyra-Perez proved in [29] that cat $(M \backslash\{p\}) \leq$ cat $(M)$, but it was unknown 1 whether the latter inequality was always strict. We prove here that this is not always the case: taking for $M$ a trivial homotopy boundary of the counterexample of Iwase to the Ganea conjecture (18), we prove

Proposition 13. There exists a simply-connected closed manifold $M$ such that for $p \in M$

$$
\operatorname{cat}(M)=\operatorname{cat}(M \backslash\{p\})=\mathrm{Cl}(M)=\mathrm{Cl}(M \backslash\{p\})=2 .
$$

This result contrasts with the behavior of the rational LS-category. Indeed if we denote by $\operatorname{cat}_{0}$ (resp. $\mathrm{Cl}_{0}$ ) the category (resp. the cone-length) of the rationalization of a space, then for every simply-connected closed manifold $M$ we have $\operatorname{cat}_{0}(M \backslash\{p\})=\operatorname{cat}_{0}(M)-1(\underline{14})$. The corresponding result for the rational cone length is wrong: the first author constructed in [23] an example of a manifold $M$ for which $\mathrm{Cl}_{0}(M \backslash\{p\})=\mathrm{Cl}_{0}(M)$, and this manifold also was obtained as a trivial homotopy boundary.

\footnotetext{
${ }^{1}$ Notice that Iwase also gives an example in [19] of a closed manifold $N$ such that $\operatorname{cat}(N \backslash\{p\})=$ cat $(N)$.
} 
Plan of the article. In the second section we set the notation used in the paper, and we recall Spanier-Whitehead duality and the James splitting theorem, which will be used in the statement of the main theorem. We also review in that section the generalized Whitehead product (absolute and relative), which will be used in the proof of our main theorem. In Section 3 we state our main theorems (Theorems 23 and 30 and their corollaries. Section 4 to 6 are devoted to the joined proofs of Theorems 23 and 30. In Section 7 we study embeddings up to rational homotopy and we give the proof of Theorem 7 . The proof of Proposition 13 on the cone-length and category of a manifold with a point deleted is the object of the last section.

Acknowledgment. We would like to thank Cristina Costoya for her help in the proof of Proposition 13, M. Mahowald and D. Ravenel who gave to us the stable homotopy theory ingredients of Example 29] in Section 3.3 and M. Arkowitz for giving us references on the generalized Whitehead product. The first author would like to thank Vincent Lemaitre and CERN for time to time hospitality and UMR "AGAT" at Université de Lille 1 for permanent hospitality. The third author would like to thank the Freie Universität Berlin for its hospitality during the time when this article was prepared.

\section{Spanier-Whitehead duality, generalized Whitehead products, AND JAMES SPLITTING}

In this section we do the following:

1. We fix the notation used in this paper.

2. We define the notion of coduality related to the Spanier-Whitehead duality.

3. We recall the definition of the generalized Whitehead products (GWP) and of the relative GWP, and some of their properties.

4. We recall the properties of the James splitting homotopy equivalence $h$ : $\Sigma \Omega \Sigma B \simeq \Sigma \bigvee_{k=1}^{\infty} B^{\wedge k}$ and the associated notion of James-Hopf invariants.

We will also state and prove many relations between all these notions.

The only notions presented in this section and needed for understanding the statement of our main theorem (in Section 3) are the following:

- the definition of a coduality (Definition [15] and Proposition [16) and of an $\mathrm{m}$ representative of a dual class (Definition 17), and

- the homotopy equivalence $\tilde{h}: \Sigma \Omega \Sigma B \simeq \Sigma B \wedge(\Omega \Sigma B)^{+}$defined at (5) and related to the James splitting.

The other notions and results of this section will be needed in the proof of our main theorem.

2.1. Notation. Here is some notation that we will use throughout the paper. We work in the category of well pointed spaces having the homotopy type of a CWcomplex. We write $*$ for the base point and the constant map. The identity map on a set is denoted by the set itself, e.g. $X: X \stackrel{\equiv}{\rightarrow}$, or sometimes id. More generally, any obvious inclusion map is denoted by the subset, e.g. $X: X \hookrightarrow X \vee Y$.

If $X$ is a space, then $C X:=X \times[0,1] / X \times\{0\}$ is the unreduced cone over $X$ and we have the obvious inclusion $X \cong X \times\{1\} \subset C X$. If $f: X \rightarrow Y$ is a map, then $C\langle f\rangle: C X \rightarrow C Y$ is the extension of that map on the cones. The suspension of $X$ is the quotient $\Sigma X=C X / X$. 
If $f: X \rightarrow Y$ is a map, we denote by $f_{\#}: \pi_{*}(X) \rightarrow \pi_{*}(Y)$ the induced map between homotopy groups. If $A$ is a subspace of $X$, we denote by $\iota: \pi_{*}(X) \rightarrow$ $\pi_{*}(X, A)$ the canonical map.

The suspension isomorphism in cohomology is denoted by

$$
\sigma: \tilde{H}^{*}(X) \stackrel{\cong}{\rightrightarrows} \tilde{H}^{*+1}(\Sigma X), \quad x \mapsto \sigma(x),
$$

and similarly in homology.

When coefficients are taken in a field $\mathbb{K}$ we have Künneth isomorphisms

$$
\begin{gathered}
\times: H^{*}(X) \otimes H^{*}(Y) \stackrel{\cong}{\rightrightarrows} H^{*}(X \times Y), \quad x \otimes y \mapsto x \times y, \\
\wedge: \tilde{H}^{*}(X) \otimes \tilde{H}^{*}(Y) \stackrel{\cong}{\rightrightarrows} \tilde{H}^{*}(X \wedge Y), \quad x \otimes y \mapsto x \wedge y .
\end{gathered}
$$

The join of two spaces is defined by

$$
X * Y:=C X \times Y \cup_{X \times Y} X \times C Y .
$$

We will use without comment the usual natural homotopy equivalence

$$
\Sigma(X \wedge Y) \simeq X * Y
$$

(41, Lemma X.8.15]), which gives an isomorphism $\tilde{H}^{*}(\Sigma(X \wedge Y)) \cong \tilde{H}^{*}(X * Y)$. Also we will compose with the canonical homeomorphism $\Sigma X \wedge Y \cong X \wedge \Sigma Y$ without writing it. For $x \in \tilde{H}^{*}(X)$ and $y \in \tilde{H}^{*}(Y)$ we have $\sigma(x \wedge y)= \pm(\sigma x) \wedge y= \pm x \wedge \sigma y$.

Maps $f: X \rightarrow Z$ and $g: Y \rightarrow Z$ induce a canonical map $(f, g): X \vee Y \rightarrow Z$. More generally if $f_{i}: X_{i} \rightarrow Z$ are maps then we have an induced map $\left(f_{i}\right)_{i}: \bigvee_{i} X_{i} \rightarrow Z$. On the other hand, if $f^{\prime}: X^{\prime} \rightarrow Z^{\prime}$ is another map, we have the map $f \vee f^{\prime}: X \vee X^{\prime} \rightarrow$ $Z \vee Z^{\prime}$. In other words, since $Z$ denotes the inclusion map $Z \hookrightarrow Z \vee Z^{\prime}$ and similarly for $Z^{\prime}$, we have $f \vee f^{\prime}=\left(Z \circ f, Z^{\prime} \circ f^{\prime}\right)$.

The adjoint of a map $f: \Sigma X \rightarrow Z$ is denoted by $f^{\natural}: X \rightarrow \Omega Z$. We write $\eta_{X}: X \rightarrow \Omega \Sigma X$ for the adjoint of the identity map on $\Sigma X$.

The restriction of a map $f: X \rightarrow Y$ to a subspace $A \subset X$ is denoted by $\left.f\right|_{A}$.

2.2. Spanier-Whitehead duality. We introduce in this section the notion of a coduality related to the Spanier-Whitehead duality. We define also the $m$ representative of a dual class (Definition [17) The latter notion will be an essential ingredient in the statement of our main theorem.

The notion of $n$-duality was introduced by Spanier and Whitehead, and in the following form by Spanier ([33]):

Definition 14. An $n$-duality between two finite CW-complexes $A$ and $A^{*}$ is a map $\alpha^{\prime}: A \wedge A^{*} \rightarrow S^{n}$ such that if $\left[S^{n}\right] \in H^{n}\left(S^{n} ; \mathbb{Z}\right)$ is a generator, then the reduced slant product induces for each $k$ an isomorphism

$$
\tilde{H}_{k}(A ; \mathbb{Z}) \stackrel{\cong}{\rightrightarrows} \tilde{H}^{n-k}\left(A^{*} ; \mathbb{Z}\right), \quad a \mapsto \alpha^{\prime *}\left(\left[S^{n}\right]\right) / a .
$$

Lemma 5.1 of [33] asserts that if $A \simeq K \subset S^{n+1}$ and if $A^{*}$ has the homotopy type of the complement $S^{n+1} \backslash K$ with $A$ and $A^{*}$ both connected, then there exists an $n$-duality between $A$ and $A^{*}$.

From the definition of the slant product and Künneth isomorphism we get that if $\alpha^{\prime}: A \wedge A^{*} \rightarrow S^{n}$ is an $n$-duality, then for each field of coefficients $\mathbb{K}$ there exist homogeneous bases $\left\{a_{i}\right\}$ of $\tilde{H}^{*}(A ; \mathbb{K})$ and $\left\{a_{i}^{*}\right\}$ of $\tilde{H}^{*}\left(A^{*} ; \mathbb{K}\right)$ such that $\alpha^{\prime *}\left(\left[S^{n}\right]\right)=$ $\sum_{i} a_{i} \wedge a_{i}^{*}$. This motivates the following dual definition. 
Definition 15. An $n$-coduality between two finite $\mathrm{CW}$-complexes $A$ and $A^{*}$ is a map $\alpha: S^{n} \rightarrow A \wedge A^{*}$ such that for each field of coefficients $\mathbb{K}$ there exist homogeneous bases $\left\{a_{i}\right\}$ of $\tilde{H}^{*}(A ; \mathbb{K})$ and $\left\{a_{i}^{*}\right\}$ of $\tilde{H}^{*}\left(A^{*} ; \mathbb{K}\right)$ such that $\alpha^{*}\left(a_{i} \wedge a_{j}^{*}\right)=\delta_{i j}\left[S^{n}\right]$, where $\delta_{i j}$ is the Kronecker symbol and $\left[S^{n}\right] \in H^{n}\left(S^{n} ; \mathbb{K}\right)$ is a generator.

Important examples of codualities are given by the following:

Proposition 16. (0) If $\alpha: S^{n} \rightarrow A \wedge A^{*}$ is an n-coduality, then $\Sigma \alpha: S^{n+1} \rightarrow$ $\Sigma A \wedge A^{*}$ is an $(n+1)$-coduality.

(1) If $A \simeq K \subset S^{n+1}$ is an h-embedding, then there exists an $(n+1)$-coduality between $\Sigma A$ and $A^{*}:=S^{n+1} \backslash K$. If moreover the $h$-embedding $A \stackrel{h}{\subset} S^{n+1}$ is the suspension of some h-embedding $A \stackrel{h}{\subset} S^{n}$, then $A^{*}$ has the homotopy type of a suspension.

(2) If $A=\bigvee_{i=1}^{r} S^{a_{i}}$ is a finite wedge of spheres and if $n \geq \operatorname{dim} A=\max \left\{a_{i}\right\}$, then there exists an $n$-coduality between $A$ and $A^{*}:=\bigvee_{i=1}^{r} S^{n-a_{i}}$. If moreover $n>\operatorname{dim} A$, then $A^{*}$ is a suspension.

Observe that an h-embedding $A \subset S^{n+1}$ gives rise to an $n$-duality but only to an $(n+1)$-coduality. Klein ([22] Theorem 7.3) proves the converse of statement (1) when $A$ and $A^{*}$ are suspensions. The above proposition is part of folklore, but we did not find a proof in the literature; therefore we kindly provide the reader with one.

Proof of Proposition 16. (0) This is an immediate application of the definition of a coduality and of the formulas $\sigma\left(a_{i} \wedge a_{j}^{*}\right)= \pm a_{i} \wedge \sigma a_{j}^{*}$. (Note that by a change of base we can replace each $\delta_{i j}$ in Definition 15 by any nonzero multiple, like $\pm \delta_{i j}$. We will use this observation without any further comment.)

(1) Let $N$ be a regular neighborhood of $K$ in $S^{n+1}$ and set $N^{*}=\overline{S^{n+1} \backslash N}$. Then $N$ and $N^{*}$ are compact manifolds with a common boundary $\partial N=\partial N^{*}$, and we have a push-out

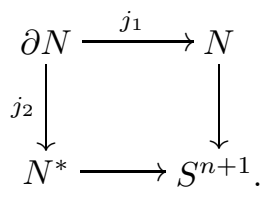

A Mayer-Vietoris argument gives isomorphisms $\tilde{H}^{k}(N) \oplus \tilde{H}^{k}\left(N^{*}\right) \cong \tilde{H}^{k}(\partial N)$ in degree $k<n$. By Poincaré duality there exist homogeneous bases $\left\{a_{i}\right\}$ and $\left\{a_{i}^{*}\right\}$ of $\tilde{H}^{k}(N)$ and $\tilde{H}^{k}\left(N^{*}\right)$ such that $j_{1}^{*}\left(a_{i}\right) \cup j_{2}^{*}\left(a_{j}^{*}\right)=\delta_{i j}[\partial N]$.

Recall that the join $N * N^{*}$ is the homotopy push-out of the projections $N \stackrel{\mathrm{pr}_{1}}{\longleftarrow}$ $N \times N^{*} \stackrel{\mathrm{pr}_{2}}{\rightarrow} N^{*}$. Therefore we have the following homotopy commutative cube 
where the top and bottom faces are homotopy push-outs:

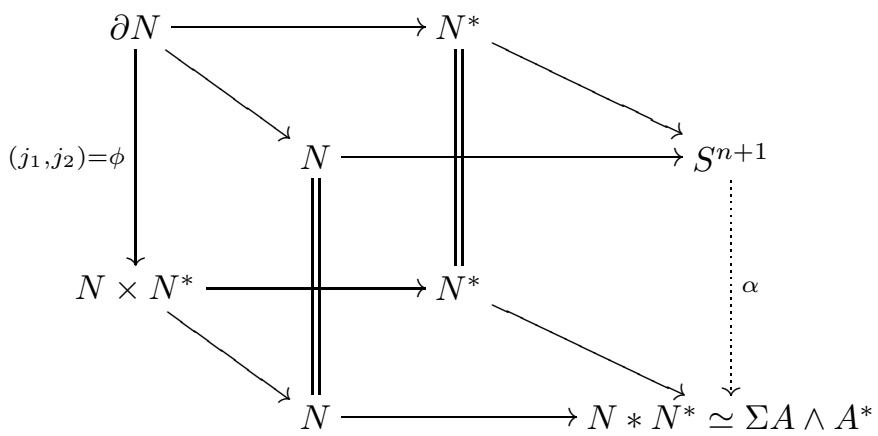

where the map $\alpha: S^{n+1} \rightarrow N * N^{*} \simeq \Sigma A \wedge A^{*}$ is induced by the universal property of the homotopy pushout. By a Mayer-Vietoris argument we get the following diagram:

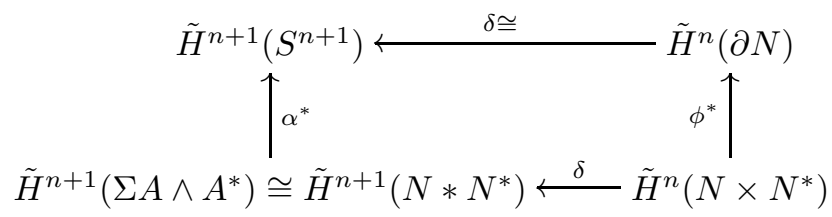

and $\delta\left(a_{i} \times a_{j}^{*}\right)=\sigma\left(a_{i}\right) \wedge a_{i}^{*}$. Since $j_{1}^{*}\left(a_{i}\right) \cup j_{2}^{*}\left(a_{j}^{*}\right)=\delta_{i j}[\partial N]$ we get that

$$
\phi^{*}\left(a_{i} \times a_{j}^{*}\right)=\delta_{i j}[\partial N]
$$

and the latter equation combined with the above diagram shows that

$$
\alpha^{*}\left(\sigma\left(a_{i}\right) \wedge a_{j}^{*}\right)=\delta_{i j}\left[S^{n+1}\right]
$$

Therefore $\alpha$ is a coduality, which establishes the first part of (1). The second part is an immediate consequence of $(0)$.

We prove (2). For $1 \leq i \leq r$, let $h_{i}: S^{n} \rightarrow S^{a_{i}} \wedge S^{n-a_{i}}$ be a homeomorphism and let $\alpha_{i} \in \pi_{n}\left(A \wedge A^{*}\right)$ be the composite of $h_{i}$ with the inclusion $S^{a_{i}} \wedge S^{n-a_{i}} \hookrightarrow A \wedge A^{*}$. Then $\alpha=\sum_{i=1}^{r} \alpha_{i}$ is an $n$-coduality.

We recall now that dualities and codualities induce Spanier-Whitehead isomorphisms between groups of stable homotopy classes. The group of stable homotopy classes between a finite CW-complex $X$ and a space $Y$ is

$$
\{X, Y\}:=\lim _{n \rightarrow \infty}\left[\Sigma^{n} X, \Sigma^{n} Y\right] .
$$

Here we only describe the isomorphisms induced by codualities.

Let $\alpha: S^{m} \rightarrow A^{*} \wedge A$ and $\beta: S^{m} \rightarrow B \wedge B^{*}$ be $m$-codualities. We have two Spanier-Whitehead homomorphisms

$$
\begin{gathered}
\alpha_{A, Z}:\{A, Z \wedge B\} \rightarrow\left\{S^{m}, Z \wedge B \wedge A^{*}\right\}, \\
\beta_{B^{*}, Z}:\left\{B^{*}, Z \wedge A^{*}\right\} \rightarrow\left\{S^{m}, Z \wedge B \wedge A^{*}\right\} .
\end{gathered}
$$

The homomorphism $\alpha_{A, Z}$ is defined as follows: if $f: \Sigma^{k} A \rightarrow \Sigma^{k}(Z \wedge B)$ is a representative of some stable homotopy class $\phi \in\{A, Z \wedge B\}$, then $\alpha_{A, Z}(\phi)=\{g\}$, where $g$ is the composite

$$
\Sigma^{k} S^{m} \stackrel{\Sigma^{k} \alpha}{\rightarrow} \Sigma^{k} A \wedge A^{*} \stackrel{f \wedge A^{*}}{\rightarrow} \Sigma^{k} Z \wedge B \wedge A^{*}
$$


The homomorphism $\beta_{B^{*}, Z}$ is defined in the same manner. It is well known (see [38, $14.19 \mathrm{seq}]$ ) that these homomorphisms are isomorphisms if $Z$ is a finite CWcomplex. The same is true if the suspension of $Z$ is a wedge of finite $\mathrm{CW}$-complexes like $Z=\Omega \Sigma B$ when $B$ is a finite $C W$-complex. For any such space $Z$ we obtain a Spanier-Whitehead duality isomorphism

$$
\mathcal{D}:=\left(\beta_{B^{*}, Z}\right)^{-1} \circ \alpha_{A, Z}:\{A, Z \wedge B\} \stackrel{\cong}{\longrightarrow}\left\{B^{*}, Z \wedge A^{*}\right\} .
$$

We will say that the image $\mathcal{D}(\{f\})$ of a class $\{f\}$ under the isomorphism $\mathcal{D}$ is the dual class of $\{f\}$. In the particular case $Z=S^{0}$, the dual class $\mathcal{D}(\{f\}) \in\left\{B^{*}, A^{*}\right\}$ of $\{f\} \in\{A, B\}$ is also called the $S$-dual of the map $f$.

The next definition will be essential in the statement of our main theorem.

Definition 17. Let $f: A \rightarrow Z \wedge B$ be a map, let $\alpha: S^{m} \rightarrow A^{*} \wedge A$ and $\beta: S^{m} \rightarrow$ $B \wedge B^{*}$ be $m$-codualities, and let $\mathcal{D}$ be the associated Spanier-Whitehead duality as above. We say that the dual class $\mathcal{D}(\{f\}) \in\left\{B^{*}, Z \wedge A^{*}\right\}$ of $\{f\}$ admits an $m$ representative if there exists a map $\rho: B^{*} \rightarrow Z \wedge A^{*}$ making the following diagram homotopy commutative:

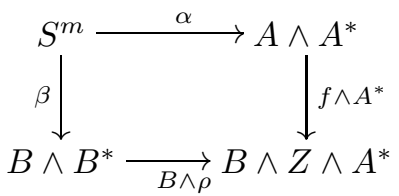

Notice that $\{\rho\}=\mathcal{D}(\{f\})$; therefore, the stable class $\mathcal{D}(\{f\})$ desuspends to $\left[B^{*}, Z \wedge A^{*}\right]$, but the latter condition is in general not sufficient to guarantee that $\mathcal{D}(\{f\})$ admits an $m$-representative in the sense of Definition 17.

2.3. Generalized Whitehead products. In this section we recall the definition of generalized Whitehead products (GWP) due to Arkowitz and we give a relative version of GWP (which was first defined in 1] and which can also be found for example in [6]). We will also recall some properties of this GWP and a classical relation between Whitehead products, coduality and Poincaré duality (Proposition 19])

Arkowitz ([2]) considers the universal GWP

$$
\overline{w_{U, V}}: U * V \simeq \Sigma(U \wedge V) \rightarrow \Sigma U \vee \Sigma V,
$$

which is defined as follows. Let $C_{-} U:=C U$ be a cone over $U$, and let $C V$ be a cone over $V$. By definition $U * V=U \times C V \cup_{U \times V} C_{-} U \times V$. Consider the two projections

$$
\begin{array}{lll}
\chi_{U}: & C_{-} U \rightarrow & \Sigma U:=C_{-} U / U, \\
\chi_{V}: & C V \rightarrow & \Sigma V:=C V / V .
\end{array}
$$

The universal GWP $\overline{w_{U, V}}$ is represented by the map

$$
h: U * V \rightarrow \Sigma U \vee \Sigma V,\left\{\begin{aligned}
&(u, y) \mapsto\left(*, \chi_{V}(y)\right) \text { if } u \in U, y \in C V, \\
&(x, v) \mapsto\left(\chi_{U}(x), *\right) \text { if } x \in C_{-} U, v \in V .
\end{aligned}\right.
$$

With this universal GWP we can define the Whitehead product of maps as follows. Let $f \in[\Sigma U, X]$ and $g \in[\Sigma V, X]$ be two maps. Their generalized Whitehead product $[f, g] \in[\Sigma U \wedge V, X]$ is defined as the composite

$$
\Sigma U \wedge V \stackrel{\overline{w_{U, V}}}{\rightarrow} \Sigma U \vee \Sigma V \stackrel{(f, g)}{\rightarrow} X
$$


As a special case, $[\Sigma U, \Sigma V]=\overline{w_{U, V}}$, where we denote by $\Sigma U$ and $\Sigma V$ the inclusions of each term into the wedge $\Sigma U \vee \Sigma V$ (the context will always be clear enough to prevent confusion between this Whitehead product $[\Sigma U, \Sigma V]$ and the set of homotopy classes of maps from $\Sigma U$ to $\Sigma V$.)

It is well known (15, sequence (9), p. 302]) that the inclusion of the homotopy fiber of $\Sigma U \vee \Sigma V \hookrightarrow \Sigma U \times \Sigma V$ is a map

$$
w_{U, V}: \Sigma \Omega \Sigma U \wedge \Omega \Sigma V \rightarrow \Sigma U \vee \Sigma V .
$$

Moreover, by [15. Lemma 5.1], the following diagram is homotopy commutative:

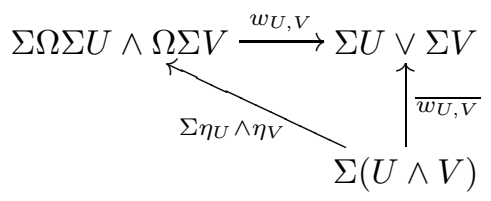

where $\eta_{U}$ and $\eta_{V}$ are the units of adjunction.

We will consider also the map $w_{U, V}^{\prime}$ defined as the composite

$$
w_{U, V}^{\prime}: \Sigma \Omega \Sigma U \wedge V \stackrel{\Sigma \Omega \Sigma U \wedge \eta_{V}}{\longrightarrow} \Sigma \Omega \Sigma U \wedge \Omega \Sigma V \stackrel{w_{U, V}}{\longrightarrow} \Sigma U \vee \Sigma V .
$$

Obviously $\overline{w_{U, V}}=w_{U, V}^{\prime} \circ \Sigma\left(\eta_{U} \wedge V\right)$.

We next give some useful formulas and a relation between the universal GWP and the cup product.

Lemma 18. Let $f: \Sigma U \rightarrow \Sigma X$ and $g: \Sigma V \rightarrow \Sigma Y$ be two maps, and denote by $\Sigma X$ and $\Sigma Y$ the inclusions of each term of the wedge $\Sigma X \vee \Sigma Y$. Then:

(i) $[\Sigma X \circ f, \Sigma Y \circ g]=(f \vee g) \overline{w_{U, V}}=w_{X, Y} \Sigma\left(f^{\natural} \wedge g^{\natural}\right)$;

(ii) if $g$ is a suspension then $(f \vee g) \overline{w_{U, V}}=w_{X, Y}^{\prime}\left(f^{\natural} \wedge g\right)$;

(iii) if $f$ and $g$ are both suspensions then $(f \vee g) \overline{w_{U, V}}=\overline{w_{X, Y}}\left(\Sigma^{-1} f \wedge g\right)$.

Proof. Formula (i) of the lemma is an immediate consequence of the following homotopy commutative diagram:

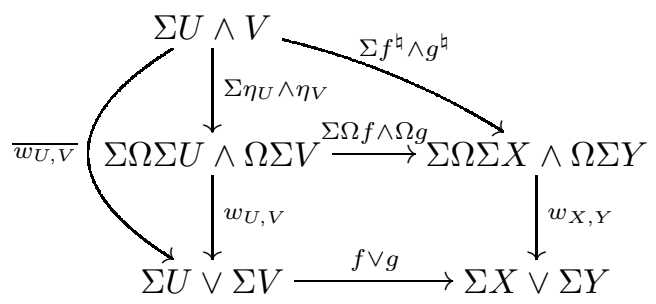

Formulas (ii) and (iii) are proved similarly, using the fact that if $g$ is a suspension then $\Sigma g^{\natural}=\left(\Sigma \eta_{V}\right) \circ g$. [22]):

The following result is very close to a result of Klein (compare to Thm. 4.5 of

Proposition 19. Let $\alpha: S^{m-1} \rightarrow \Sigma U \wedge U^{*}$ be a coduality. The homotopy cofibre $\left(\Sigma U \vee \Sigma U^{*}\right) \cup_{\Lambda} e^{m}$ of the composite $\Lambda: S^{m-1} \stackrel{\alpha}{\rightarrow} \Sigma U \wedge U^{*} \stackrel{\text { w }_{U, U^{*}}}{\rightarrow} \Sigma U \vee \Sigma U^{*}$ is a Poincaré duality space. More precisely, let $\mathbb{K}$ be any field and $\left\{u_{i}\right\},\left\{u_{i}^{*}\right\}$ be homogeneous bases of $\tilde{H}^{*}(U ; \mathbb{K})$ and $\tilde{H}^{*}\left(U^{*} ; \mathbb{K}\right)$ such that $\alpha^{*}\left(\left(\sigma u_{i}\right) \wedge u_{j}^{*}\right)=\delta_{i j}\left[S^{m-1}\right]$. 
Then in $H^{*}\left(\left(\Sigma U \vee \Sigma U^{*}\right) \cup_{\Lambda} e^{m} ; \mathbb{K}\right)$ we have

$$
\begin{aligned}
\sigma u_{i} \cup \sigma u_{j}^{*} & =\delta_{i j}\left[e^{m}\right], \\
\sigma u_{i} \cup \sigma u_{j} & =\sigma u_{i}^{*} \cup \sigma u_{j}^{*}=0,
\end{aligned}
$$

where $\left[e^{m}\right]$ is the pullback of the cohomology class $\left[S^{m}\right]$ by the pinching map $\left(\Sigma U \vee \Sigma U^{*}\right) \cup_{\Lambda} e^{m} \rightarrow S^{m}$.

Proof. Consider the following diagram, in which each horizontal line is a cofibration sequence (by Theorem 4.2 of [2] for the second line) and where $g$ is the induced application between the cofibres:

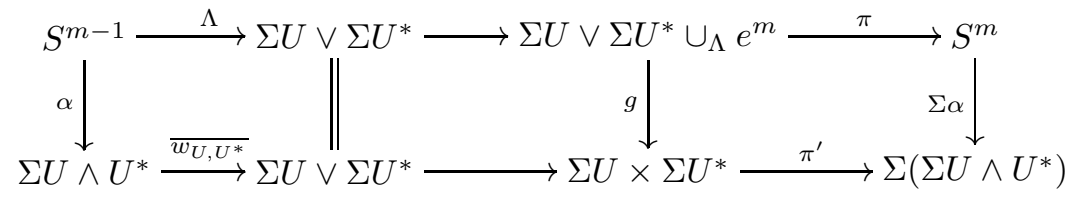

In $H^{*}\left(\Sigma U \vee \Sigma U^{*} \cup_{\Lambda} e^{m}\right)$ we thus have:

$$
\begin{aligned}
\sigma u_{i} \cup \sigma u_{j}^{*} & =H^{*}(g)\left(\sigma u_{i} \times \sigma u_{j}^{*}\right) \\
& =H^{*}(g) H^{*}\left(\pi^{\prime}\right)\left(\sigma u_{i} \wedge \sigma u_{j}^{*}\right) \\
& = \pm H^{*}(\pi) H^{*}(\Sigma \alpha)\left(\sigma\left(\sigma u_{i} \wedge u_{j}^{*}\right)\right) \\
& = \pm H^{*}(\pi)\left(\delta_{i j}\left[S^{m}\right]\right) \\
& = \pm \delta_{i j}\left[e^{m}\right] .
\end{aligned}
$$

The formulas $\sigma u_{i} \cup \sigma u_{j}=\sigma u_{i}^{*} \cup \sigma u_{j}^{*}=0$ are proved by looking at the retractions of $\Sigma U$ and $\Sigma U^{*}$ out of $\Sigma U \vee \Sigma U^{*} \cup_{\Lambda} e^{m}$.

We have recalled the definition of the universal GWP

$$
\overline{w_{U, V}} \in[\Sigma U \wedge V, \Sigma U \vee \Sigma V] .
$$

There exists also a universal relative $G W P$, which is a relative homotopy class

$$
\overline{W_{U, V}} \in[(C \Sigma U \wedge V, \Sigma U \wedge V),((C \Sigma U) \vee \Sigma V, \Sigma U \vee \Sigma V)]
$$

This universal relative GWP is for example defined in [6, 0.3] as the unique relative homotopy class whose image by the boundary map

$$
\partial:[(C \Sigma U \wedge V, \Sigma U \wedge V),((C \Sigma U) \vee \Sigma V, \Sigma U \vee \Sigma V)] \longrightarrow[\Sigma U \wedge V, \Sigma U \vee \Sigma V]
$$

is $\partial\left(\overline{W_{U, V}}\right)=\overline{w_{U, V}}$.

In order to prove some properties that we need of this universal relative GWP we will give a more explicit description of it. Recall the explicit description $h$ of $\overline{w_{U, V}}$ given at (1). Let $C_{+} U$ be a second cone over $U$ and set $\Sigma^{\prime} U=C_{+} U \cup_{U} C_{-} U$. We have a homotopy equivalence

$$
\varsigma: \Sigma^{\prime} U \stackrel{\simeq}{\longrightarrow} \Sigma U
$$

defined by $\left.\varsigma\right|_{C_{+} U}=*$ and $\left.\varsigma\right|_{C_{-} U}=\chi_{U}$.

It is not difficult to construct a natural (in $U$ and $V$ ) homotopy equivalence

$$
\phi: C(U * V) \stackrel{\simeq}{\rightrightarrows} C_{+} U \times C V \cup_{C_{+} U \times V} C \Sigma^{\prime} U \times V
$$


whose restriction to $U * V$ is the inclusion map

$\left.\phi\right|_{U * V}: U * V=U \times C V \cup_{U \times V} C_{-} U \times V\left(C_{+} U \times C V \cup_{C_{+} U \times V} C \Sigma^{\prime} U \times V\right.$.

We define $H: C(U * V) \rightarrow C \Sigma U \vee \Sigma V$ as the composite $H^{\prime} \circ \phi$, where

$$
H^{\prime}: C_{+} U \times C V \cup_{C_{+} U \times V} C \Sigma^{\prime} U \times V \rightarrow(C \Sigma U) \vee \Sigma V
$$

is defined by

$$
\begin{cases}H^{\prime}(x, y)=\left(*, \chi_{V}(y)\right) & \text { if } x \in C_{+} U, y \in C V \\ H^{\prime}(z, v)=(C\langle\varsigma\rangle(z), *) & \text { if } z \in C \Sigma^{\prime} U, v \in V\end{cases}
$$

As $\left.H\right|_{U \times C V \cup_{U \times V} C_{-} U \times V}=h$, we thus obtain a pair of maps $(H, h)$. The relative homotopy class of this pair is denoted by

$$
\overline{W_{U, V}} \in[(C(U * V), U * V),((C \Sigma U) \vee \Sigma V, \Sigma U \vee \Sigma V)],
$$

and it is called the universal relative GWP. Obviously $\partial\left(\overline{W_{U, V}}\right)=\overline{w_{U, V}}$, which proves that our definition agrees with that of Baues [6].

Let $X$ be a subspace of $Y$ and denote by $i: X \hookrightarrow Y$ the inclusion. We define now a relative version of the GWP:

$$
\begin{aligned}
{[-,-]:[(C \Sigma U, \Sigma U),(Y, X)] \times[\Sigma V, X] } & \rightarrow[(C(U * V), U * V),(Y, X)], \\
((F, f), g) & \mapsto[(F, f), g] .
\end{aligned}
$$

If $(F, f) \in[(C \Sigma U, \Sigma U),(Y, X)]$ and $g \in[\Sigma V, X]$, then the relative GWP is by definition the relative homotopy class in $[(C(U * V), U * V),(Y, X)]$ determined by the following diagram:

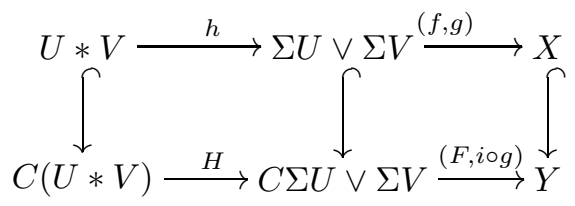

Clearly, this relative GWP satisfies $\partial([(F, f), g])=[f, g]$, where $\partial$ is the boundary map

$$
\partial:[(C(U * V), U * V),(Y, X)] \rightarrow[U * V, X], \quad(K, k) \mapsto k .
$$

When no confusion is possible we will write $[F, g]$ instead of $[(F, f), g]$. We also set $[g, F]:=-[F, g]$.

We finish this section with a relation between the relative universal GWP $\left(\overline{W_{U, V}}, \overline{w_{U, V}}\right)$ and the absolute universal GWP $\overline{w_{\Sigma U, V}}$. The following lemma will be used in the proof of Proposition 36, which is a step in the proof of our main theorem. Note that point (i) of this lemma is also proved in [6. Corollary 3.1.12], but we give another proof for the sake of completeness.

Lemma 20. Let $U, V$ be finite $C W$-complexes and consider the canonical projections $\xi$ and $\Xi$ obtained by shrinking $\Sigma U$ to the base point:

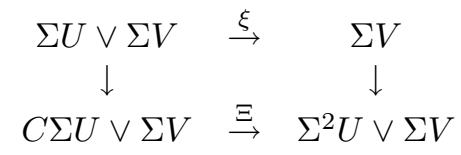


Denote by $\Gamma$ the following canonical map between sets of homotopy classes

$$
\begin{aligned}
\Gamma:\left[\Sigma(U * V), \Sigma^{2} U \vee \Sigma V\right] & \cong\left[(C(U * V), U * V),\left(\Sigma^{2} U \vee \Sigma V, *\right)\right] \\
& \stackrel{\iota}{\longrightarrow}\left[(C(U * V), U * V),\left(\Sigma^{2} U \vee \Sigma V, \Sigma V\right)\right] .
\end{aligned}
$$

Then there exists a natural homotopy equivalence $\theta: \Sigma(U * V) \stackrel{\simeq}{\rightarrow}(\Sigma U) * V$ such that

(i) $\Gamma\left(\overline{w_{\Sigma U, V}} \circ \theta\right)=\left(\Xi \circ \overline{W_{U, V}}, \xi \circ \overline{w_{U, V}}\right)$ in $\left[(C(U * V), U * V),\left(\Sigma^{2} U \vee \Sigma V, \Sigma V\right)\right]$, and

(ii) for $u \in \tilde{H}^{*}(U)$ and $v \in \tilde{H}^{*}(V)$ we have $H^{*}(\theta)(\sigma((\sigma u) \wedge v))= \pm \sigma^{2}(u \wedge v)$.

Proof. Consider two copies $C_{+}(U * V)$ and $C_{-}(U * V)$ of the cone $C(U * V)$. Recall the natural homotopy equivalence

$$
\phi: C_{+}(U * V) \stackrel{\simeq}{\longrightarrow} C_{+} U \times C V \cup_{C_{+} U \times V} C \Sigma^{\prime} U \times V,
$$

whose restriction to $U * V$ is the inclusion map. There exists also a natural homeomorphism

$$
\psi: C_{-}(U * V) \stackrel{\cong}{\longrightarrow} C_{-} U \times C V,
$$

whose restriction to $U * V$ is the identity map $U * V=U \times C V \cup_{U \times V} C_{-} U \times V$.

Denote by $I_{-}(U * V):=(U * V) \times[0,1]$ the cylinder on $U * V$, and consider the two standard inclusion maps $i_{0}, i_{1}: U * V \rightarrow I_{-}(U * V)$. We have the canonical projection

$$
\pi: I_{-}(U * V) \longrightarrow I_{-}(U * V) / i_{1}(U * V)=C_{-}(U * V)
$$

and $\pi \circ i_{0}$ is the usual inclusion of $U * V$ into the cone.

We have the following strictly commutative diagram:

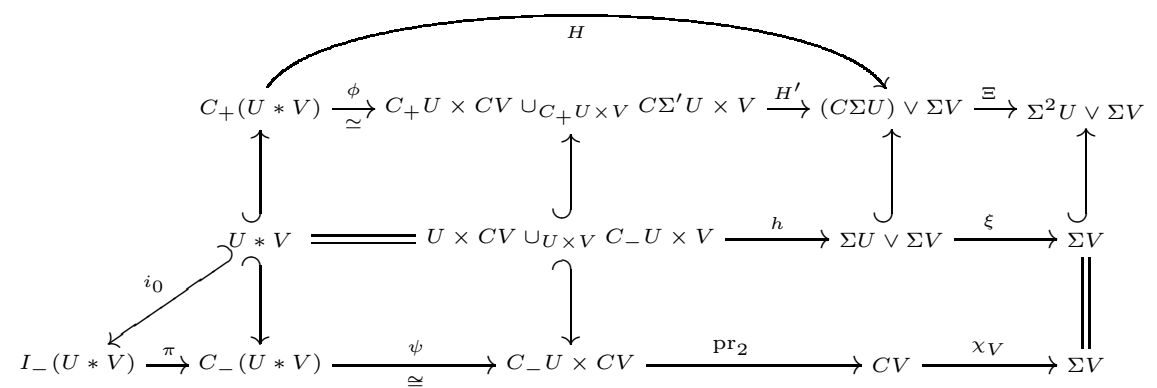

Consider the obvious natural homeomorphism

$$
\Theta: C(U * V) \stackrel{\cong}{\longrightarrow} C_{+}(U * V) \cup_{i_{0}(U * V)} I_{-}(U * V),
$$

whose restriction to $U * V$ is the inclusion $i_{1}$. Taking push-outs on the left part of Diagram (3) induces maps $F=\left(\Xi \circ H, \chi_{V} \circ \mathrm{pr}_{2} \circ \psi \circ \pi\right)$ and $\bar{F}=\left(\Xi \circ H, \chi_{V} \circ \mathrm{pr}_{2} \circ \psi\right)$ in the following commutative diagram where vertical maps are the projections on 
the quotients by $U * V$ and $\bar{\Theta}$ is the homeomorphism induced by $\Theta$ :

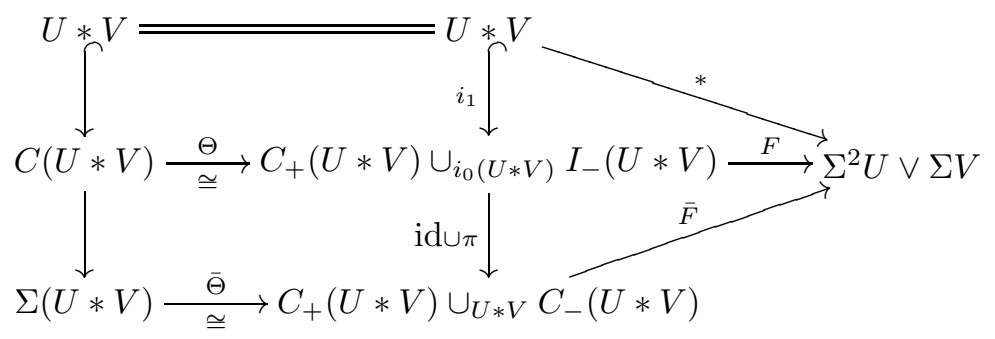

Since the restriction $\left.F\right|_{I_{-}(U * V)}$ takes value into $\Sigma V$ and $\left.F\right|_{C_{+}(U * V)}=\Xi \circ H$, it is clear that the pairs $(F \circ \Theta, *)$ and $(\Xi \circ H, \xi \circ h)$ represent the same relative homotopy class in $\left[(C(U * V), U * V),\left(\Sigma^{2} U \vee \Sigma V, \Sigma V\right)\right]$.

We have also a homotopy equivalence

$$
\begin{aligned}
& C_{+}(U * V) \cup_{U * V} C_{-}(U * V) \\
& \qquad \begin{array}{l}
\not \cup \psi \\
\simeq
\end{array} \\
& \left.\quad C_{+} U \times C V \cup_{C_{+} U \times V} C \Sigma^{\prime} U \times V\right) \cup_{U * V} C_{-} U \times C V C V \cup_{\Sigma^{\prime} U \times V} C \Sigma^{\prime} U \times V \\
& \quad=\left(\Sigma^{\prime} U\right) * V .
\end{aligned}
$$

Using the definitions of $H^{\prime}$ and $h$, it is straightforward to check that the map

$$
\bar{F}: C_{+}(U * V) \cup_{U * V} C_{-}(U * V) \rightarrow \Sigma^{2} U \vee \Sigma V
$$

is exactly the composite

$$
\begin{aligned}
& C_{+}(U * V) \cup_{U * V} C_{-}(U * V) \stackrel{\phi \cup \psi}{\cong}\left(\Sigma^{\prime} U\right) * V \stackrel{\overline{w_{\Sigma^{\prime} U, V}}}{\longrightarrow} \Sigma \Sigma^{\prime} U \vee \Sigma U \\
& \stackrel{\Sigma \varsigma \vee \Sigma V}{\simeq} \Sigma^{2} U \vee \Sigma V .
\end{aligned}
$$

In other words, $\bar{F}=[\Sigma \varsigma, \Sigma V] \circ(\phi \cup \psi)$.

Define the homotopy equivalence $\theta: \Sigma(U * V) \stackrel{\simeq}{\rightarrow}(\Sigma U) * V$ as the composite

$$
\Sigma(U * V) \stackrel{\bar{\Theta}}{\simeq} C_{+}(U * V) \cup_{U * V} C_{-}(U * V) \stackrel{\phi \cup \psi}{\simeq}\left(\Sigma^{\prime} U\right) * V \stackrel{\varsigma * V}{\simeq}(\Sigma U) * V .
$$

From Lemma 18 (iii) we compute that $[\Sigma \varsigma, \Sigma V]=\overline{w_{\Sigma U, V}} \circ(\varsigma * V)$. Therefore $\bar{F} \circ \bar{\Theta}=\overline{w_{\Sigma U, V}} \circ \theta$. In conclusion we have

$$
\Gamma\left(\overline{w_{\Sigma U, V}} \circ \theta\right)=\Gamma(\bar{F} \circ \bar{\Theta})=(F \circ \Theta, *)
$$

and, as we have seen before, the latter represents the same relative homotopy class as $(\Xi \circ H, \xi \circ h)$ in $\left[(C(U * V), U * V),\left(\Sigma^{2} U \vee \Sigma V, \Sigma V\right)\right]$. This completes the proof of (i).

We prove formula (ii). Let $u \in \tilde{H}^{p}(U)$ and $v \in \tilde{H}^{q}(V)$ be homogeneous cohomology classes. Denote by $U_{p}$ the $p$-skeleton of the CW-complex $U$ and consider the inclusion map $i: U_{p} \rightarrow U$. Set $u^{\prime}:=H^{*}(i)(u) \in H^{p}\left(U_{p}\right)$. There exists a shrinking map $\pi: U_{p} \rightarrow S^{p}$ to a sphere such that $\tilde{H}^{*}(\pi)\left(\left[S^{p}\right]\right)=u^{\prime}$, where $\left[S^{p}\right] \in \tilde{H}^{p}\left(S^{p}\right)$ is the fundamental class. Similarly we have maps

$$
S^{p} \stackrel{\pi^{\prime}}{\longleftarrow} V_{q} \stackrel{i^{\prime}}{\longrightarrow} V
$$


such that $\tilde{H}^{*}\left(\pi^{\prime}\right)\left(\left[S^{q}\right]\right)=v^{\prime}:=\tilde{H}^{*}\left(i^{\prime}\right)(v)$. Set $r=p+q$. For each couple $(k, l)$ such that $k+l=r$, consider the $k$-skeleton $U_{k} \hookrightarrow U$ and the $l$-skeleton $V_{l} \hookrightarrow V$. Since $\theta$ is natural, the following diagram is commutative:

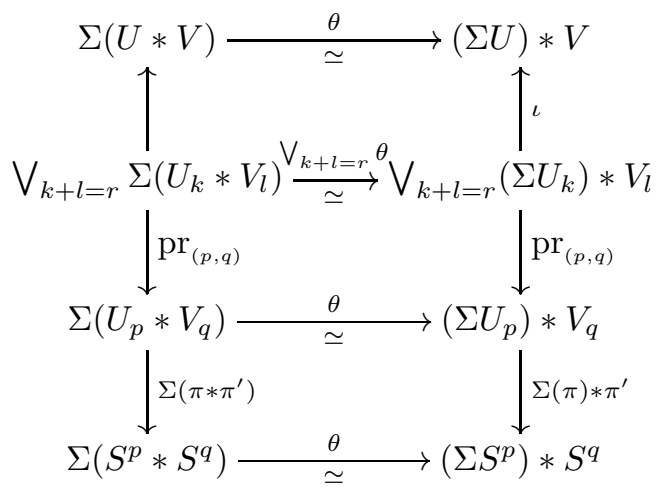

The bottom map is a homotopy equivalence between homotopy spheres. Therefore it induces a map of degree \pm 1 in cohomology. Thus

$$
\tilde{H}^{*}(\theta)\left(\sigma^{2}\left(u^{\prime} \wedge v^{\prime}\right)\right)= \pm \sigma\left(\left(\sigma u^{\prime}\right) \wedge v^{\prime}\right) .
$$

It is easy to check that $\tilde{H}^{r+2}(\iota)$ is injective, using the fact that the $r+2$-skeleton of $(\Sigma U) * V$ is

$$
((\Sigma U) * V)_{r+2}=\cup_{k+l=r}\left(\Sigma U_{k}\right) * V_{l}
$$

Therefore

$$
\tilde{H}^{*}(\theta)\left(\sigma^{2}(u \wedge v)\right)= \pm \sigma((\sigma u) \wedge v)
$$

2.4. James splitting and James-Hopf invariants. In this section we recall the James splitting homotopy equivalence and we introduce an associated homotopy equivalence $\tilde{h}$ (formula (5) ) which will be an important ingredient in the statement of our main theorem. We will also prove a relation between the Whitehead product and the James equivalence.

If $B$ is a pointed connected CW-complex, then the James splitting theorem (see 20]) gives a homotopy equivalence

$$
h: \Sigma \Omega \Sigma B \stackrel{\simeq}{\rightarrow} \Sigma \bigvee_{k=1}^{\infty} B^{\wedge k}
$$

We recall a construction of that homotopy equivalence. Denote by $J(B)$ the James reduced product which is the topological free monoid generated by $B$ ([41], Chapter VII.2, or [20]), and identify $J(B)$ with $\Omega \Sigma B$ by the usual homotopy equivalence. The space $J B$ is filtered by $*=J^{0} B \subset J^{1} B \subset J^{2} B \subset \cdots \subset J B$, where $J^{m} B$ consists of the words of length less than $m$ in the monoid. We have maps

$$
g_{k}: J(B) \rightarrow J\left(B^{\wedge k}\right)
$$

defined by $g_{k}\left(b_{1} \cdot \cdots \cdot b_{m}\right)=\prod_{1 \leq i_{1}<\ldots<i_{k} \leq m}\left(b_{i_{1}} \wedge \cdots \wedge b_{i_{k}}\right)$, where the order of the factors is the right lexicographical order (41], page 334, or [7], II, (2.3)).

The James-Hopf invariants

$$
\gamma^{k}:[\Sigma U, \Sigma B] \rightarrow\left[\Sigma U, \Sigma B^{\wedge k}\right]
$$


are by definition characterized by $\left(\gamma^{k}(f)\right)^{\natural}=g_{k} \circ\left(f^{\natural}\right)$ for $f: \Sigma U \rightarrow \Sigma B$. Notice that $\gamma^{1}(f)=f$. As a consequence of $\left(\gamma^{k}(f)\right)^{\natural}=g_{k} \circ\left(f^{\natural}\right)$ we have $g_{k}=\left(\gamma^{k}\left(\operatorname{ev}_{\Sigma B}\right)\right)^{\natural}$, where $\operatorname{ev}_{\Sigma B}: \Sigma \Omega(\Sigma B) \rightarrow \Sigma B$ is the evaluation map.

Consider the inclusions $j_{k}: \Sigma B^{\wedge k} \hookrightarrow \Sigma \bigvee_{i=1}^{\infty} B^{\wedge i}$. Then the James splitting homotopy equivalence is defined as $h:=\sum_{k=1}^{\infty} j_{k} \gamma^{k}\left(\operatorname{ev}_{\Sigma B}\right)$. This sum is finite at each point $x \in \Sigma J(B) \simeq \Sigma \Omega \Sigma B$, because for $k>m$ the restriction $\gamma^{k}\left(\operatorname{ev}_{\Sigma B}\right) \mid \Sigma J^{m}(B)$ is the constant map $*$ since $g_{k} \mid J^{m}(B)=*$.

From the James equivalence, we define two other homotopy equivalences. Denote by $(\Omega \Sigma B)^{+}=\Omega \Sigma B \vee S^{0}$ the space $\Omega \Sigma B$ with an adjoined point and the original basepoint. We define the map

$$
h^{+}: \Sigma\left((\Omega \Sigma B)^{+}\right) \rightarrow \Sigma \bigvee_{l=0}^{\infty} B^{\wedge l}
$$

by

$$
\left\{\begin{array}{l}
h^{+} \mid \Sigma(\Omega \Sigma B)=h, \\
h^{+} \mid \Sigma S^{0} \text { is the inclusion through } \Sigma B^{\wedge 0}=\Sigma S^{0} .
\end{array}\right.
$$

This map $h^{+}$is a homotopy equivalence. Also, the homotopy equivalences $h$ : $\Sigma \Omega \Sigma B \stackrel{\simeq}{\rightarrow} \Sigma \bigvee_{l=1}^{\infty} B^{\wedge k}$ and $h^{+}: \Sigma(\Omega \Sigma B)^{+} \stackrel{\simeq}{\rightarrow} \Sigma \bigvee_{l=0}^{\infty} B^{\wedge k}$ give rise to a homotopy equivalence

$$
\left(B \wedge h^{+}\right)^{-1} \circ h: \Sigma \Omega \Sigma B \stackrel{\simeq \Sigma}{\longrightarrow} \bigvee_{k=1}^{\infty} B^{\wedge k} \simeq B \wedge \Sigma(\Omega \Sigma B)^{+} \cong \Sigma B \wedge(\Omega \Sigma B)^{+}
$$

which we will denote by

$$
\tilde{h}: \Sigma \Omega \Sigma B \stackrel{\simeq}{\rightarrow} \Sigma B \wedge(\Omega \Sigma B)^{+} .
$$

We recall a well-known property of James-Hopf invariants. Let $f: \Sigma U \rightarrow \Sigma B$ be a map. From $\left(\gamma^{k}(f)\right)^{\natural}=g_{k} \circ\left(f^{\natural}\right)$ we deduce that $\gamma^{k}(f)=\gamma^{k}\left(\operatorname{ev}_{\Sigma B}\right) \circ \Sigma f^{\natural}$. In particular, if $g: \Sigma V \rightarrow \Sigma U$ is a co-H-map, then $\Sigma(f \circ g)^{\natural} \simeq\left(\Sigma f^{\natural}\right) \circ g$ and we have that

$$
\text { if } g \text { is a co-H-map then } \gamma^{k}(f \circ g)=\gamma^{k}(f) \circ g \text {. }
$$

Notice also that if $\operatorname{dim}(U)<\infty$ then $\gamma^{k}(f)$ is trivial for any $k$ sufficiently large, and so we have $\sum_{k=1}^{\infty} j_{k} \gamma^{k}(f)=h \circ \Sigma f^{\natural}$.

The James equivalence permits us also to express the map $w_{U, V}^{\prime}$ defined at (2) in the previous section in terms of iterated Whitehead products. More precisely, for $k \geq 0$ we define maps

$$
\bar{w}_{k}: \Sigma U^{\wedge k} \wedge V \rightarrow \Sigma U \vee \Sigma V
$$

by $\bar{w}_{0}=\Sigma V: \Sigma V \hookrightarrow \Sigma U \vee \Sigma V$ and $\bar{w}_{k+1}=\left[\Sigma U, \bar{w}_{k}\right]=\left(\Sigma U, \bar{w}_{k}\right) \bar{w}_{U, U^{\wedge k} \wedge V}$. In other words, $\bar{w}_{k}=[\Sigma U,[\Sigma U,[\cdots[\Sigma U, \Sigma V] \cdots]]$ with $k$ factors $\Sigma U$. Then we have:

Lemma 21. Suppose that $U$ is a finite $C W$-complex and that $V$ is a $C W$-complex and a co-H-space. Then the following diagram is homotopy commutative:

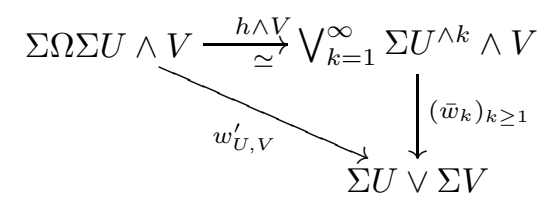


Proof of Lemma 21] Using the fact that $\left(\operatorname{ev}_{\Sigma U}\right)^{\natural}$ is the identity map $\Omega \Sigma U$ and that $(\Sigma V)^{\natural}$ is the unit of adjunction $\eta_{V}: V \rightarrow \Omega \Sigma V$, we have by Lemma 18 (i) that the following diagram is homotopy commutative:

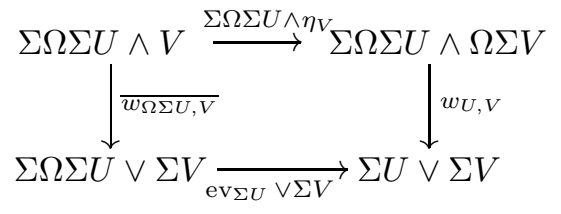

Therefore

(8) $w_{U, V}^{\prime} \stackrel{\text { def }}{=} w_{U, V} \circ\left(\Sigma \Omega \Sigma U \wedge \eta_{V}\right)=\left(\operatorname{ev}_{\Sigma U} \vee \Sigma V\right) \circ \overline{w_{\Omega \Sigma U, V}}=\left[\Sigma U \circ \operatorname{ev}_{\Sigma U}, \Sigma V\right]$.

To continue the proof we recall the following formula, which is proved in [7, Prop. II 3.4, page 51] or [4]:

Proposition 22. Suppose that $X$ is a co-H-space, that $Y$ is a finite dimensional $C W$-complex, that $Z_{0}$ is a $C W$-complex and that we have maps $\Sigma X \stackrel{\zeta}{\rightarrow} Z_{0}$ and $\Sigma Y \stackrel{\theta}{\rightarrow} \Sigma U \stackrel{\eta}{\rightarrow} Z_{0}$. Then

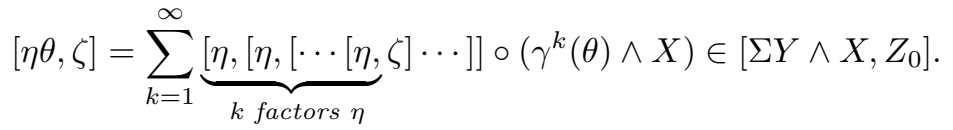

We apply this formula for $Z_{0}=\Sigma U \vee \Sigma V, X=V, Y=\Omega \Sigma U, \zeta$ being the inclusion $\Sigma V: \Sigma V \hookrightarrow Z_{0}, \theta=\operatorname{ev}_{\Sigma U}: \Sigma \Omega \Sigma U \rightarrow \Sigma U$, and $\eta$ being the inclusion $\Sigma U: \Sigma U \hookrightarrow Z_{0}$. We obtain

$$
\left[\Sigma U \circ \operatorname{ev}_{\Sigma U}, \Sigma V\right]=\sum_{k=1}^{\infty} \underbrace{[\Sigma U,[\Sigma U,[\cdots[\Sigma U,}_{k \text { factors } \Sigma U}, \Sigma V] \cdots]] \circ\left(\gamma^{k}\left(\operatorname{ev}_{\Sigma U}\right) \wedge V\right) .
$$

Note that unlike the required condition in Proposition 22 , the space $Y=\Omega \Sigma U$ is not finite dimensional. In fact, this hypothesis is needed in [7, Proposition II.3.4] only to guarantee that the infinite sum in the formula is finite at each point. This is the case in formula (91), since $\gamma^{k}\left(\operatorname{ev}_{\Sigma U}\right) \mid \Sigma J^{m}(U)=*$ for $k>m$. In fact the proof of Proposition 22 in [7] passes exactly through the proof of equation (9) above (see equation (2), p. 52 in [7]). Alternatively, one can also use the fact that $\bigoplus\left[A_{i}, K\right] \cong\left[\bigvee A_{i}, K\right]$ in order to prove the formula in our case.

Formula (8) then becomes

$$
\begin{aligned}
w_{U, V}^{\prime} & =\left[\Sigma U \circ \operatorname{ev}_{\Sigma U}, \Sigma V\right] \\
& =\sum_{k=1}^{\infty} \underbrace{[\Sigma U,[\Sigma U,[\cdots[\Sigma U,}_{k \text { factors } \Sigma U}, \Sigma V] \cdots]] \circ\left(\gamma^{k}\left(\operatorname{ev}_{\Sigma U}\right) \wedge V\right) \\
& =\sum_{k=1}^{\infty} \bar{w}_{k} \circ\left(\gamma^{k}\left(\operatorname{ev}_{\Sigma U}\right) \wedge V\right) \\
& =\left(\bar{w}_{k}\right)_{k \geq 1} \circ\left(\sum_{k=1}^{\infty} \gamma^{k}\left(\operatorname{ev}_{\Sigma U}\right) \wedge V\right) \\
& =\left(\bar{w}_{k}\right)_{k \geq 1}(h \wedge V) \quad \text { by the definition of } h,
\end{aligned}
$$

and this finishes the proof of Lemma 21. 


\section{StATEMENT OF THE MAIN THEOREM AND SOME COROLlARIES}

In this section we state our main theorem, and some corollaries and examples are developed. We use the notions related to the Spanier-Whitehead duality recalled in Section 2.2 and the homotopy equivalence $\tilde{h}: \Sigma \Omega \Sigma B \cong \Sigma \cong \wedge(\Omega \Sigma B)^{+}$defined in Section 2.4]

Let $f: \Sigma A \rightarrow \Sigma B$ be a map. Consider the map $\Sigma f^{\natural}: \Sigma A \rightarrow \Sigma \Omega \Sigma B$. We thus have $\left\{\tilde{h} \Sigma f^{\natural}\right\} \in\left\{\Sigma A, \Sigma B \wedge(\Omega \Sigma B)^{+}\right\}$. Recall from Section 2.2 that if $\alpha: S^{n-2} \rightarrow$ $\Sigma A \wedge A^{*}$ and $\beta: S^{n-2} \rightarrow \Sigma B \wedge B^{*}$ are codualities, we have a Spanier-Whitehead duality isomorphism

$$
\mathcal{D}:\left\{\Sigma A,(\Omega \Sigma B)^{+} \wedge \Sigma B\right\} \stackrel{\cong}{\rightrightarrows}\left\{B^{*}, A^{*} \wedge(\Omega \Sigma B)^{+}\right\} .
$$

Recall also that we say (Definition [17) that the class $\mathcal{D}\left(\left\{\tilde{h} \Sigma f^{\natural}\right\}\right)$ admits an $(n-2)$ representative iff there exists a map

$$
\rho: B^{*} \rightarrow(\Omega \Sigma B)^{+} \wedge A^{*}
$$

making the following diagram homotopy commutative:

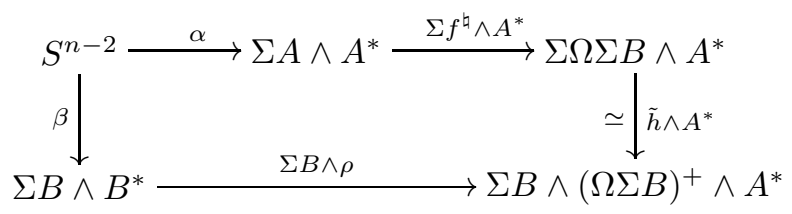

We now state our main theorem:

Theorem 23. Let $A$ and $B$ be two connected finite $C W$-complexes and let $n \geq 6$. Let $f: \Sigma A \rightarrow \Sigma B$ be a map, and denote by $X$ its homotopy cofibre. Assume that $\tilde{H}^{*}(f ; \mathbb{Z})=0$, and suppose given two $(n-2)$-codualities $\alpha: S^{n-2} \rightarrow \Sigma A \wedge A^{*}$ and $\beta: S^{n-2} \rightarrow \Sigma B \wedge B^{*}$ such that $A^{*}$ and $B^{*}$ are suspensions.

If the class $\mathcal{D}\left(\left\{\tilde{h} \Sigma f^{\natural}\right\}\right) \in\left\{B^{*},(\Omega \Sigma B)^{+} \wedge A^{*}\right\}$ admits an $(n-2)$ representative, then there exists a tractable h-embedding $X \stackrel{h}{\subset} S^{n+1}$.

Remarks. 1. By Proposition 16 the existence of an $(n-2)$-coduality $\alpha$ such that $A^{*}$ is a suspension is guaranteed if any of the following holds:

- $A$ is a finite wedge of spheres of dimension $\leq n-4$,

- $A$ h-embeds in $S^{n-4}$, or

- $\Sigma A$ h-embeds in $S^{n-3}$,

and similar conditions on $B$ implies the existence of a coduality $\beta$ such that $B^{*}$ is a suspension.

2. When $\Sigma A=*$, the theorem above is a corollary of Theorem 7.3 of [22].

3. If $\rho: B^{*} \rightarrow(\Omega \Sigma B)^{+} \wedge A^{*}$ is an $(n-2)$-representative of $\mathcal{D}\left(\left\{\tilde{h} \Sigma f^{\natural}\right\}\right)$, then we have a homotopy commutative diagram as in diagram 100 .

From the composition of this diagram with the projection $\Sigma B \wedge(\Omega \Sigma B)^{+} \wedge A^{*} \rightarrow$ $\Sigma B \wedge A^{*}$ we deduce that the projection of $\rho$ on $A^{*}$, i.e., the composites $B^{*} \stackrel{\rho}{\rightarrow}$ $(\Omega \Sigma B)^{+} \wedge A^{*}=(\Omega \Sigma B) \wedge A^{*} \vee A^{*} \rightarrow A^{*}$, is an $(n-2)$-representative of the S-dual $\mathcal{D}(\{f\}) \in\left\{B^{*}, A^{*}\right\}$ of $f$.

We defer the proof of this theorem to the next section, and we now consider Theorem 23 in some special situations. 
3.1. h-embeddings of two-cell complexes. Let $f: S^{q-1} \rightarrow S^{p}$ be a map and $f^{\natural}: S^{q-2} \rightarrow \Omega S^{p}$ be the adjoint map. Recall from (4) that the James-Hopf invariants $\gamma^{k}(f) \in \pi_{q-1}\left(S^{k(p-1)+1}\right)$ of $f$ are the composites

$$
S^{q-1} \stackrel{\Sigma f^{\natural}}{\rightarrow} \Sigma \Omega S^{p} \stackrel{h \simeq}{\longrightarrow} \bigvee_{j=1}^{\infty} S^{j(p-1)+1} \stackrel{\operatorname{pr}_{k}}{\rightarrow} S^{k(p-1)+1}
$$

In particular, $\gamma^{1}(f)=f$. Then we have the following statement, which makes Corollary 5 of the Introduction precise:

Corollary 24. Let $q \geq p \geq 2$ and $n \geq q+2$. Let $f \in \pi_{q-1}\left(S^{p}\right)$ and set $X=$ $S^{p} \cup_{f} e^{q}$. If, for each $k \geq 1, \Sigma^{n-q-1} \gamma^{k}(f)$ desuspends $p$ times to some $\rho^{k} \in$ $\pi_{n-p-2}\left(S^{k(p-1)+n-q-p}\right)$, then there exists a tractable h-embedding $X \stackrel{h}{\subset} S^{n+1}$.

Proof. In order to apply Theorem 23 we choose the obvious codualities $\alpha: S^{n-2}=$ $S^{q-1} \wedge S^{n-q-1}$ and $\beta: S^{n-2}=S^{p} \wedge S^{n-p-2}$. Let

$$
\iota_{k}: S^{(k(p-1)+n-q-p} \rightarrow \bigvee_{i=1}^{\infty} S^{i(p-1)+n-q-p}
$$

be the inclusion. Set $\tilde{\rho}=\sum_{k \geq 1} \iota_{k} \rho^{k}$ (this sum is finite since for reason of dimension the map $\rho^{k}$ is trivial for $k$ sufficiently large). As $\Sigma^{p} \rho^{k}=\Sigma^{n-q-1} \gamma^{k}(f)$ for any $k \geq 1$, we have

$$
\Sigma^{p} \tilde{\rho}=\sum_{k \geq 1} \Sigma^{n-q-1}\left(\Sigma^{p} \iota_{k}\right) \gamma^{k}(f)=\Sigma^{n-q-1} h \Sigma f^{\natural} .
$$

Observe now that

$$
\tilde{\rho} \in \pi_{n-p-2}\left(\bigvee_{i=1}^{\infty} S^{i(p-1)+n-q-p}\right)=\pi_{n-p-2}\left(\bigvee_{i=1}^{\infty} S^{(i-1)(p-1)+1+n-q-2}\right) .
$$

We finally compose $\tilde{\rho}$ with the homotopy equivalence

$$
\Sigma^{n-q-2}\left(h^{+}\right)^{-1}: S^{n-q-2} \wedge \bigvee_{i=1}^{\infty} S^{(i-1)(p-1)+1)} \rightarrow S^{n-q-2} \wedge \Sigma\left(\Omega S^{p}\right)^{+},
$$

and the resulting map $\rho: S^{n-p-2} \rightarrow\left(\Omega S^{p}\right)^{+} \wedge S^{n-q-1}$ makes homotopy commutative the diagram (10). Therefore there is a tractable h-embedding $X \stackrel{h}{\subset} S^{n+1}$.

Let us use Corollary 24 to exhibit an example of an h-embedding whose existence does not follow from the theorem of Connolly-Williams (Theorem 3 in the Introduction).

Example 25. Consider the composite $f: S^{29} \stackrel{w}{\rightarrow} S^{15} \stackrel{\eta}{\rightarrow} S^{8}$, where $w$ is the Whitehead product $\left[S^{15}, S^{15}\right]$ and $\eta$ is the Hopf map. It follows from the EHP sequence together with Theorem XII-2.4 of [41] that $w=\left[S^{15}, S^{15}\right]$ is both essential and a suspension. Since $w$ is a suspension, formula (6) of Section 2.4 implies that $\gamma^{k}(f)=\gamma^{k}(\eta) \circ w, k \geq 1$. Therefore $\gamma^{1}(f)=f, \gamma^{2}(f)=w$ and $\gamma^{k}(f)=0$ for $k \geq 3$. Since $w$ is a Whitehead product, $\Sigma w=0$, and it follows that $\Sigma \gamma^{1}(f) \in \pi_{30}\left(S^{9}\right)$ and $\Sigma \gamma^{2}(f) \in \pi_{30}\left(S^{16}\right)$ are both zero. These two maps then desuspend 8 times and, by Corollary 24] $S^{8} \cup_{f} e^{30}$ h-embeds in $S^{33}$. 
The previous corollary permits also, in the theorem of Connolly-Williams (for $p \geq 5$ ), a widening of the range of codimensions for which the desuspendability of a certain suspension of $f$ ensures the existence of an h-embedding:

Corollary 26. Let $q \geq p \geq 2, n \geq q+2$, and $f \in \pi_{q-1}\left(S^{p}\right)$. Suppose that $n \geq 2 q-3 p+3$. If $\Sigma^{n-q-1} f$ desuspends $p$ times, then there exists a tractable h-embedding $S^{p} \cup_{f} e^{q} \stackrel{h}{\subset} S^{n+1}$.

Proof. The condition $n \geq 2 q-3 p+3$ together with the Freudenthal theorem implies that $\Sigma^{n-q-1} \gamma^{2}(f)$ desuspends $p$ times, and so do the maps $\Sigma^{n-q-1} \gamma^{k}(f)$ for $k \geq$ 3. Therefore the hypothesis of Corollary 24 reduces to the $p$-desuspendability of $\Sigma^{n-q-1} \gamma^{1}(f)=\Sigma^{n-q-1} f$.

As we wrote in the introduction, we lose in this corollary one dimension in comparison to the result of Connolly-Williams, but we gain the fact that our hembedding is tractable.

3.2. When $X$ is a two-stage $\mathbf{C W}$-complex. We now specialise our main theorem to two-stage CW-complexes. For this we need the following:

Definition 27. Let $A=\bigvee_{i=1}^{p} S^{a_{i}}$ and $B=\bigvee_{j=1}^{q} S^{b_{j}}$ be two connected finite wedges of spheres. The $j$ th projected global James-Hopf invariant of a map $f: \Sigma A$ $\rightarrow \Sigma B$ is the composite, denoted by $\gamma_{[j]}^{*}(f)$,

$$
\Sigma A \stackrel{\Sigma f^{\natural}}{\rightarrow} \Sigma \Omega \Sigma B \stackrel{h \simeq}{\rightarrow} \Sigma\left(\bigvee_{k=1}^{\infty} B^{\wedge k}\right) \stackrel{\pi_{j}}{\rightarrow} \Sigma\left(\bigvee_{k=1}^{\infty} S^{b_{j}} \wedge B^{\wedge k-1}\right)
$$

where $\pi_{j}$ is the composite

$$
\pi_{j}: \Sigma\left(\bigvee_{k=1}^{\infty} B^{\wedge k}\right)=\Sigma\left(\bigvee_{l=1}^{q} \bigvee_{k=1}^{\infty} S^{b_{l}} \wedge B^{\wedge k-1}\right) \stackrel{\mathrm{pr}_{j}}{\rightarrow} \Sigma\left(\bigvee_{k=1}^{\infty} S^{b_{j}} \wedge B^{\wedge k-1}\right)
$$

With this definition our criterion for two-stage CW-complexes becomes:

Corollary 28. Let $A=\bigvee_{i=1}^{p} S^{a_{i}}$ and $B=\bigvee_{j=1}^{q} S^{b_{j}}$ be finite wedges of connected spheres, let $f=\left(f_{i}\right)_{1 \leq i \leq p}: \Sigma A \rightarrow \Sigma B$ be a map, and denote by $X$ its homotopy cofibre. Let $n$ be an integer such that $n \geq \max (\operatorname{dim} \Sigma A, \operatorname{dim} \Sigma B, 3)+3$.

If for each $1 \leq i \leq p$ and each $1 \leq j \leq q$ the iterated suspension of the $j$ th projected global James-Hopf invariant

$$
\Sigma^{n-a_{i}-3} \gamma_{[j]}^{*}\left(f_{i}\right) \in \pi_{n-2}\left(\Sigma^{n-a_{i}+b_{j}-2} \bigvee_{l=0}^{\infty} B^{\wedge l}\right)
$$

desuspends $\left(b_{j}+1\right)$ times, then there exists a tractable h-embedding of $X$ in $S^{n+1}$.

Proof. Set $A^{*}=\bigvee_{i=1}^{p} S^{n-a_{i}-3}$. This is a suspension because $n-a_{i}-3 \geq 1$. Consider the evident homeomorphisms $\alpha_{i}: S^{n-2} \rightarrow \Sigma S^{a_{i}} \wedge S^{n-a_{i}-3}$ and set $\alpha=$ $\sum_{i=1}^{p} \alpha_{i}: S^{n-2} \rightarrow \Sigma A \wedge A^{*}$. The map $\alpha$ is a coduality. Similarly set $B^{*}=$ $\bigvee_{j=1}^{p} S^{n-b_{j}-3}$ and consider the evident coduality $\beta: S^{n-2} \rightarrow \Sigma B \wedge B^{*}$.

Let $\rho_{j}^{i}$ be a $\left(b_{j}+1\right)$-desuspension of the map

$$
\Sigma^{n-a_{i}-3} \gamma_{[j]}^{*}\left(f_{i}\right) \in \pi_{n-2}\left(\Sigma^{n-a_{i}+b_{j}-2} \bigvee_{l=0}^{\infty} B^{\wedge l}\right)
$$


and set $\rho_{j}=\sum_{i=1}^{p}\left(h^{+} \wedge S^{n-a_{i}-2}\right) \rho_{j}^{i} \in \pi_{n-b_{j}-3}\left((\Omega \Sigma B)^{+} \wedge S^{n-a_{i}-3}\right)$. Finally set $\rho=\left(\rho_{j}\right)_{1 \leq j \leq q} \in\left[B^{*},(\Omega \Sigma B)^{+} \wedge A^{*}\right]$. An elementary computation shows that diagram (10) is commutative, and the corollary follows.

3.3. About the converse of our main theorem. Here we show that the converse of Theorem 23 fails to be true. We construct examples of two-cones $\Sigma A \stackrel{f}{\rightarrow}$ $\Sigma B \rightarrow X$ with $\tilde{H}^{*}(f ; \mathbb{Z})=0$ such that $X$ h-embeds in some $S^{n+1}$ but there is no $(n-2)$-coduality between $\Sigma B$ and any other space. Actually we construct, for an arbitrarily large integer $i$, a two-cone $X=\Sigma B \cup_{f} C \Sigma A$ which h-embeds in some $S^{n+1}$ and such that there exists no $(n+i-2)$-coduality between $\Sigma B$ and any other space.

Example 29. Let $j \geq 2$, and set $n=2^{j+1}+1$ and $i=2^{j}-2$. In the following we use the same Greek letter to name a stable homotopy class in $\pi_{k}^{S}$ and any of its representative in $\pi_{k+r}\left(S^{r}\right)$. Let $\eta_{j} \in \pi_{2^{j}}^{S}$ be the stable homotopy class defined by Mahowald [25] whose sphere of origin is $S^{2^{j}-2}$, and let $\eta \in \pi_{1}^{S}$ be the Hopf map. Set $B=S^{2^{j}-2} \cup_{\eta_{j} \circ \eta} e^{2^{j+1}}$ and $A=S^{2^{j}-1}$. Define $f: \Sigma A \rightarrow \Sigma B$ as the composite of the Hopf map with the inclusion of the bottom sphere of $\Sigma B$. The homotopy cofibre of $f$ is $X=S^{2^{j}-1} \cup_{\eta} e^{2^{j}+1} \cup_{\eta_{j} \circ \eta} e^{2^{j+1}+1} \simeq \Sigma^{2^{j}-3} \mathbb{C} P(2) \vee S^{2^{j+1}+1}$. Therefore $X \stackrel{h}{\subset} S^{n+1}$, where $n=2^{j+1}+1$. On the other hand, it is known ([26], 30]) that the sphere of origin of $\eta_{j} \circ \eta$ is $S^{2^{j}-4}$. Thus by the theorem of Connolly-Williams (Theorem 3 above) or by the main theorem of [17, we have that $\Sigma B \not \subset S^{2^{j+1}+2^{j}-3}$. By Theorem 7.3 of [22] this implies that for $i \leq 2^{j}-2$ there is no coduality $S^{n+i-2} \rightarrow \Sigma B \wedge B^{*}$.

This example shows that the converse of our main theorem does not hold. Of course in the latter example the cone decomposition of $X$ is not the natural one, and for the obvious one (i.e. where $B$ above is replaced by $S^{2^{j}-2} \vee S^{2^{j+1}}$ ), the converse of Theorem 23 holds. We do not know if the converse of our main theorem holds for a "reasonable" decomposition of a two-cone. We do even not know if the hypothesis on the Hopf invariants in Corollary 24 is a necessary condition for the existence of a tractable h-embedding of a two-cell complex.

3.4. Homotopy boundary induced by the h-embedding. Recall from the Introduction that to any h-embedding $X \simeq K \subset S^{n+1}$ is associated a Euclidean thickening obtained as a regular neighborhood of $K$ (Definition 9). We can describe the homotopy type of the boundary of the thickening induced by the h-embedding of Theorem 23.

Theorem 30. Under the same hypotheses as in Theorem 203, $X$ admits a tractable Euclidean homotopy boundary $\partial N$ of dimension $n$ whose homotopy type is described as follows:

$$
\partial N \simeq Z \cup_{\Psi} e^{n}
$$

where:

$Z=\left(\Sigma B \vee \Sigma A^{*}\right) \cup_{f} C \Sigma A \cup_{\phi} C \Sigma B^{*}$,

$\phi: \Sigma B^{*} \rightarrow \Sigma B \vee \Sigma A^{*}$ is a map determined by the $(n-2)$-representative $\rho: B^{*} \rightarrow$ $(\Omega \Sigma B)^{+} \wedge A^{*}$ of the dual class $\mathcal{D}\left(\left\{\tilde{h} \Sigma f^{\natural}\right\}\right)$,

$\Psi \in \pi_{n-1}(Z)$ is determined by $\alpha, \beta$, and $\rho$ up to some indeterminacy in the image of $\pi_{n}\left(\Sigma B \times \Sigma A^{*}, \Sigma B \vee \Sigma A^{*}\right)$ under a certain map. 
This theorem will be proved in the next section. The explicit formula for $\phi$ in terms of $\rho$ will be given in (13) in the next section, and the determination of $\Psi$ will be made in the proof of Lemma 35

When $X$ is a two-cell complex $S^{p} \cup_{f} e^{q}$ we can give a more explicit description:

Corollary 31. Let $f: S^{q-1} \rightarrow S^{p}$ be as in Corollary 24 and let

$$
\rho^{k} \in \pi_{n-p-2}\left(S^{(k(p-1)+n-q-p}\right)
$$

be the $p$-th desuspension of $\Sigma^{n-q-1} \gamma^{k}(f)$. The Euclidean homotopy boundary $\partial N$ of $X=S^{p} \cup_{f} e^{q}$ induced by the h-embedding $X \stackrel{h}{\subset} S^{n+1}$ given by Corollary 24 has the following homotopy type:

$$
\begin{aligned}
& \partial N=Z \cup_{\Psi} e^{n}, \\
& Z=S^{p} \cup_{f} e^{q} \vee S^{n-q} \cup_{\phi} e^{n-p} \cup_{\Psi} e^{n}, \\
& \phi=\sum_{k=1}^{\infty} \underbrace{\left[S^{p},\left[S^{p}, \cdots,\left[S^{p}\right.\right.\right.}_{(k-1) \text { factors } S^{p}}, S^{n-q}] \cdots]] \circ\left(\Sigma \rho^{k}\right) \in \pi_{n-p-1}\left(S^{p} \vee S^{n-q}\right), \text { and } \\
& \Psi \in \pi_{n-1}(Z) \text { is a certain lift of the sum of relative Whitehead products }
\end{aligned}
$$

$$
\left[S^{p}, e^{n-p}\right]+\left[e^{q}, S^{n-q}\right] \in \pi_{n-1}\left(Z, S^{p} \vee S^{n-q}\right) .
$$

Finally, in the stable range, we obtain a description of the trivial homotopy boundary (see Definition [11) of a simply connected finite two-cone $X=\Sigma B \cup_{f} C \Sigma A$ with $\tilde{H}^{*}(f ; \mathbb{Z})=0$. Indeed, let $X$ be such a two-cone and $n \geq 2 \operatorname{dim} X+2$. As $n \geq \max (2 \operatorname{dim}(\Sigma A)+2,2 \operatorname{dim}(\Sigma B)+2)$, there exist codualities $\alpha: S^{n-2} \rightarrow \Sigma A \wedge A^{*}$ and $\beta: S^{n-2} \rightarrow \Sigma B \wedge B^{*}$. Moreover, the Freudenthal isomorphism implies that the image of $\left\{\tilde{h} \Sigma f^{\natural}\right\}$ under the isomorphism $\mathcal{D}$ admits an $(n-2)$-representative $\rho: B^{*} \rightarrow(\Omega \Sigma B)^{+} \wedge A^{*}$. In other words, the hypotheses of Theorem 30 are fulfilled and we have:

Corollary 32. Let $A$ and $B$ be two connected finite $C W$-complexes, let $f: \Sigma A \rightarrow$ $\Sigma B$ be a map, and denote by $X$ its homotopy cofibre. Assume that $\tilde{H}^{*}(f ; \mathbb{Z})=0$, and let $n \geq 2 \operatorname{dim}(X)+2$ be an integer. Consider $(n-2)$-codualities $\alpha: S^{n-2} \rightarrow$ $\Sigma A \wedge A^{*}$ and $\beta: S^{n-2} \rightarrow \Sigma B \wedge B^{*}$. The image of $\left\{\tilde{h} \Sigma f^{\natural}\right\}$ under the SpanierWhitehead isomorphism $\mathcal{D}$ admits an $(n-2)$-representative $\rho: B^{*} \rightarrow(\Omega \Sigma B)^{+} \wedge A^{*}$, and the homotopy type of the $n$-dimensional trivial homotopy boundary of $X$ is described as follows:

$$
\mathcal{B}^{n}(X)=\left(\Sigma B \vee \Sigma A^{*}\right) \cup_{f} C \Sigma A \cup_{\phi} C \Sigma B^{*} \cup_{\Psi} e^{n},
$$

where the maps $\phi$ and $\Psi$ are determined by $\rho, \alpha$ and $\beta$ as in Theorem 30 .

Notice that by Theorem 10 of the Introduction, $\mathcal{B}^{n}(X)$ depends neither on the choice of the codualities $\alpha$ and $\beta$, nor on the $(n-2)$-representative $\rho$.

\section{Proof of the main theorem}

In this section we prove jointly Theorems 23 and 30 We use the notation of the statements of these two theorems. The proof is based on

Theorem 33 (Browder ([8], Theorem 1.1)). Suppose given a homotopy push-out

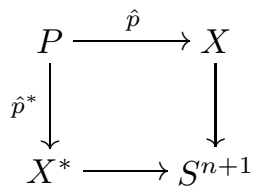


such that $n \geq 6, P$ is a simply-connected Poincaré duality space of dimension $n$, and $X$ and $X^{*}$ are simply-connected finite $C W$-complexes of dimension $\leq n$. Then there exist two compact submanifolds $N$ and $N^{*}$ of codimension 0 in $S^{n+1}$ such that $N \simeq X, N^{*} \simeq X^{*}, N \cup N^{*}=S^{n+1}$, and $N \cap N^{*}=\partial N=\partial N^{*}$ has the same homotopy type as $P$. More precisely we have a homotopy commutative diagram in which vertical arrows are homotopy equivalences:

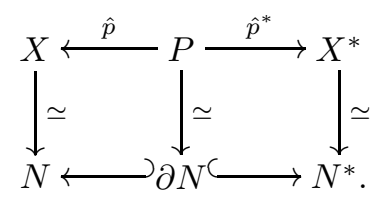

In particular $X$ h-embeds in $S^{n+1}$ and $P$ has the homotopy type of a Euclidean boundary of $X$.

This theorem of Browder is proved using surgery theory to smooth the Poincaré duality pairs $(X, P)$ and $\left(X^{*}, P\right)$.

Suppose given a map $f: \Sigma A \rightarrow \Sigma B$ and codualities $\alpha: S^{n-2} \rightarrow \Sigma A \wedge A^{*}$ and $\beta: S^{n-2} \rightarrow \Sigma B \wedge B^{*}$ that satisfies the hypotheses of Theorem 23. The idea of the proof of Theorem 23 is to construct a Poincaré duality space $P$, a space $X^{*}$ and projections $X \stackrel{\hat{p}}{\leftarrow} P \stackrel{\hat{p}^{*}}{\rightarrow} X^{*}$ such that there is a homotopy push-out like in Browder's theorem with $X=\Sigma B \cup_{f} C \Sigma A$. The space $P$ will be obtained by means of successive cone attachments to $\Sigma B \vee \Sigma A^{*}$. The final form of $P$ will be

$$
P=\Sigma B \vee \Sigma A^{*} \cup_{f} C \Sigma A \cup_{\phi} C \Sigma B^{*} \cup_{\Psi} e^{n}
$$

with $\phi \in\left[\Sigma B^{*}, \Sigma B \vee \Sigma A^{*}\right]$ and $\Psi \in \pi_{n-1}\left(\Sigma B \vee \Sigma A^{*} \cup_{f} C \Sigma A \cup_{\phi} C \Sigma B^{*}\right)$. This construction of $P$ will give the description of $\partial N$ in Theorem 30 .

In the course of the proof we will state two lemmas (34 and 35) and a proposition (36) that will be proved in Sections 5 and 6 respectively.

We first construct the map $\phi: \Sigma B^{*} \rightarrow \Sigma B \vee \Sigma A^{*}$. By hypothesis the class $\mathcal{D}\left(\left\{\tilde{h} \Sigma f^{\natural}\right\}\right)$ admits an $(n-2)$ representative. That means that there exists a map

$$
\rho: B^{*} \rightarrow(\Omega \Sigma B)^{+} \wedge A^{*}
$$

making the following diagram homotopy commutative:

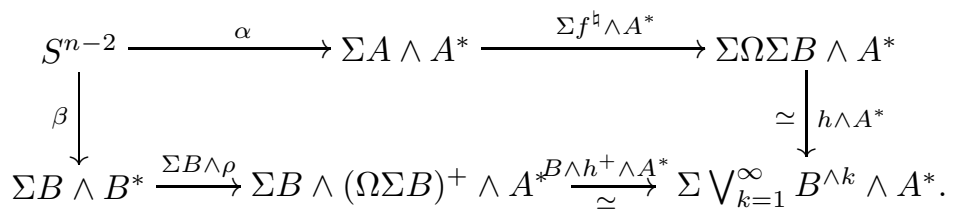

(note that the commutativity of the latter diagram is equivalent to that of Diagram (10) because by definition $\tilde{h}=\left(B \wedge h^{+}\right)^{-1} \circ h$, see Section 2.4 (15) ).

Denote by $\rho^{\prime}: B^{*} \rightarrow \Omega \Sigma B \wedge A^{*}$ and $\rho^{\prime \prime}: B^{*} \rightarrow A^{*}$ the composites of $\rho$ with the two obvious projections from $(\Omega \Sigma B)^{+} \wedge A^{*} \simeq\left(\Omega \Sigma B \wedge A^{*}\right) \vee A^{*}$. Then

$$
\Sigma \rho=\Sigma \rho^{\prime}+\Sigma \rho^{\prime \prime} \in\left[\Sigma B^{*}, \Sigma(\Omega \Sigma B)^{+} \wedge A^{*}\right] .
$$


Consider the map $w_{B, A^{*}}^{\prime}: \Sigma \Omega \Sigma B \wedge A^{*} \rightarrow \Sigma B \vee \Sigma A^{*}$ as defined in Section 2.3] (2) and define the maps

$$
\begin{aligned}
& \phi^{\prime}: \Sigma B^{*} \stackrel{\Sigma \rho^{\prime}}{\rightarrow} \Sigma \Omega \Sigma B \wedge A^{*} \stackrel{w_{B, A^{*}}^{\prime}}{\longrightarrow} \Sigma B \vee \Sigma A^{*}, \\
& \phi^{\prime \prime}: \Sigma B^{*} \stackrel{\Sigma \rho^{\prime \prime}}{\longrightarrow} \Sigma A^{*} \stackrel{\Sigma A^{*}}{\longrightarrow} \Sigma B \vee \Sigma A^{*} .
\end{aligned}
$$

We set

$$
\phi=\phi^{\prime}+\phi^{\prime \prime} \in\left[\Sigma B^{*}, \Sigma B \vee \Sigma A^{*}\right]
$$

Define the spaces

$$
\begin{aligned}
Z_{0} & =\Sigma B \vee \Sigma A^{*}, \\
Z & =Z_{0} \cup_{f} C \Sigma A \cup_{\phi} C \Sigma B^{*}, \\
Z_{+} & =Z_{0} \cup_{f} C \Sigma A, \\
Z_{-} & =Z_{0} \cup_{\phi} C \Sigma B^{*},
\end{aligned}
$$

and denote by $j_{+}: Z_{+} \hookrightarrow Z, j_{-}: Z_{-} \hookrightarrow Z$, and $j_{0}: Z_{0} \hookrightarrow Z$ the inclusion maps.

We now define a space $X^{*}$. Consider the obvious projections from $Z_{0}=\Sigma B \vee \Sigma A^{*}$

$$
\begin{array}{ll}
p_{0}: & Z_{0} \rightarrow \Sigma B, \\
p_{0}^{*}: & Z_{0} \rightarrow \Sigma A^{*} .
\end{array}
$$

Set $f^{*}=p_{0}^{*} \phi$ and

$$
X^{*}=\Sigma A^{*} \cup_{f^{*}} C \Sigma B^{*} .
$$

We want to define projections $p: Z \rightarrow X$ and $p^{*}: Z \rightarrow X^{*}$. It is clear from the definition of $\phi$ that $p_{0} \phi$ is homotopically trivial. Therefore there exists a homotopy equivalence

$$
g_{-}: Z_{-} / \Sigma A^{*} \stackrel{\simeq}{\rightarrow} \Sigma B \vee \Sigma^{2} B^{*}
$$

which extends by the identity on $C \Sigma A$ to a homotopy equivalence

$$
g: Z / \Sigma A^{*} \stackrel{\simeq}{\rightarrow} X \vee \Sigma^{2} B^{*} \text {. }
$$

On the other hand, $p_{0}^{*} f=*$. Thus we have the identity maps

$$
g_{+}^{*}: Z_{+} / \Sigma B \stackrel{\equiv}{\rightarrow} \Sigma A^{*} \vee \Sigma^{2} A
$$

and

$$
g^{*}: Z / \Sigma B \stackrel{\equiv}{\rightarrow} X^{*} \vee \Sigma^{2} A .
$$

Now define the following composites where the first maps are the obvious projections:

$$
\begin{aligned}
& q: Z \stackrel{\mathrm{pr}}{\longrightarrow} Z / \Sigma A^{*} \stackrel{g}{\simeq} X \vee \Sigma^{2} B^{*}, \\
& q^{*}: Z \stackrel{\mathrm{pr}}{\longrightarrow} Z / \Sigma B \stackrel{g^{*}}{\longrightarrow} X^{*} \vee \Sigma^{2} A .
\end{aligned}
$$

The projections $p$ and $p^{*}$ are defined as the following composites:

$$
\begin{aligned}
& p: Z \stackrel{q}{\rightarrow} X \vee \Sigma^{2} B^{*} \stackrel{\mathrm{pr}}{\rightarrow} X, \\
& p^{*}: Z \stackrel{q^{*}}{\rightarrow} X^{*} \vee \Sigma^{2} A \stackrel{\mathrm{pr}}{\rightarrow} X^{*} .
\end{aligned}
$$


The next step is to construct a map $\Psi: S^{n-1} \rightarrow Z$ such that the space $P:=$ $Z \cup_{\Psi} e^{n}$ satisfies Poincaré duality and the projections $p: Z \rightarrow X$ and $p^{*}: Z \rightarrow X^{*}$ extend to $Z \cup_{\Psi} e^{n}$.

Consider the inclusions of the cones:

$$
\begin{aligned}
F: C \Sigma A & \rightarrow Z_{0} \cup_{f} C \Sigma A=Z_{+}, \\
\Phi: C \Sigma B^{*} & \rightarrow Z_{0} \cup_{\phi} C \Sigma B^{*}=Z_{-} .
\end{aligned}
$$

The pair $(F, f) \in\left[(C \Sigma A, \Sigma A),\left(Z_{+}, Z_{0}\right)\right]$ is a relative homotopy class. Denote by $\Sigma A^{*} \in\left[\Sigma A^{*}, Z_{0}\right]$ the obvious inclusion. We can consider the relative GWP $\left[F, \Sigma A^{*}\right] \in\left[(C \Sigma A, \Sigma A),\left(Z_{+}, Z_{0}\right)\right]$ as defined in Section 2.3. Similarly we have a relative GWP $[\Sigma B, \Phi] \in\left[\left(C \Sigma B^{*}, \Sigma B^{*}\right),\left(Z_{-}, Z_{0}\right)\right]$. The coduality $\alpha: S^{n-2} \rightarrow A * A^{*}$ extends to $C\langle\alpha\rangle: D^{n-1}=C S^{n-2} \rightarrow C\left(A * A^{*}\right)$, and similarly for $\beta$. We can then define the following homotopy classes of pairs of maps (see the diagram (17) below):

$$
\begin{aligned}
& \tilde{\Omega}_{+}=\left(\left[F, \Sigma A^{*}\right] C\langle\alpha\rangle,\left[f, \Sigma A^{*}\right] \alpha\right) \in \pi_{n-1}\left(Z_{+}, Z_{0}\right), \\
& \tilde{\Omega}_{-}=([\Sigma B, \Phi] C\langle\beta\rangle,[\Sigma B, \phi] \beta) \in \pi_{n-1}\left(Z_{-}, Z_{0}\right), \\
& \Omega_{+}=\left(j_{+}\right)_{\#}\left(\tilde{\Omega} \tilde{\Omega}_{+}\right) \in \pi_{n-1}\left(Z, Z_{0}\right), \\
& \Omega_{-}=\left(j_{-}\right)_{\#}\left(\tilde{\Omega}_{-}\right) \in \pi_{n-1}\left(Z, Z_{0}\right), \\
& \Omega=\Omega_{+}-\Omega_{-} \in \pi_{n-1}\left(Z, Z_{0}\right) .
\end{aligned}
$$

The following lemma is crucial for the existence of the desired map $\Psi: S^{n-1} \rightarrow Z$, and we point out that the commutativity (up to homotopy) of the diagram (11) will play an important role in the proof of this lemma.

Lemma 34. $\left[f, \Sigma A^{*}\right] \circ \alpha=[\Sigma B, \phi] \circ \beta$ in $\pi_{n-2}\left(Z_{0}\right)$.

The proof of this lemma and also that of Lemma 35 below are deferred to the next section.

We have the following diagram in which each part is commutative by definition, or by Lemma 34 for the inner subdiagram

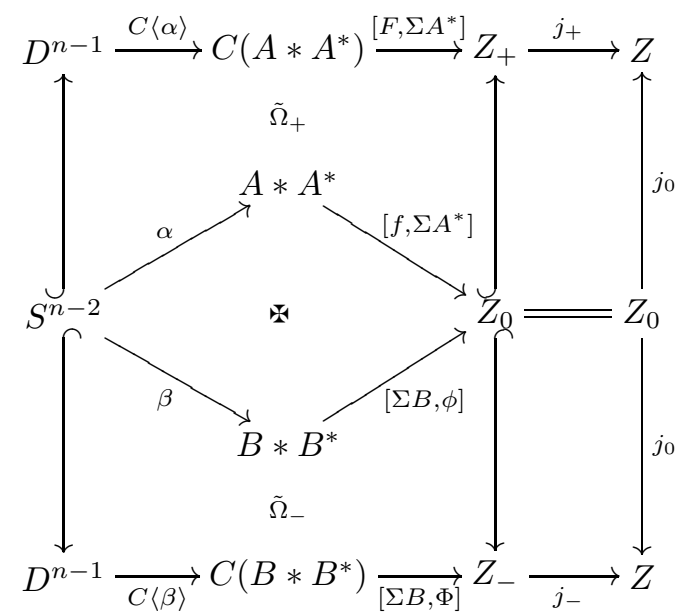

The commutativity of the subdiagram implies that the relative homotopy classes $\Omega_{+}$and $\Omega_{-}$have the same boundary in $\pi_{n-1}\left(Z_{0}\right)$. Therefore the class $\Omega=\Omega_{+}-$ $\Omega_{-} \in \pi_{n-1}\left(Z, Z_{0}\right)$ admits a lifting in $\pi_{n-1}(Z)$. The following lemma asserts that we can choose this lifting $\Psi$ of $\Omega$ such that the projections $p: Z \rightarrow X$ and $p^{*}: Z \rightarrow X^{*}$ extend to $Z \cup_{\Psi} e^{n}$. 
Lemma 35. There exists $\Psi \in \pi_{n-1}(Z)$ such that

(i) $\iota(\Psi)=\Omega$, where $\iota: \pi_{n-1}(Z) \rightarrow \pi_{n-1}\left(Z, Z_{0}\right)$ is the obvious map;

(ii) $p_{\#}(\Psi)=0$ in $\pi_{n-1}(X)$ and $p_{\#}^{*}(\Psi)=0$ in $\pi_{n-1}\left(X^{*}\right)$; and

(iii) conditions (i) and (ii) determine $\Psi$ up to some indeterminacy in the image of $\pi_{n}\left(\Sigma B \times \Sigma A^{*}, \Sigma B \vee \Sigma A^{*}\right)$ by the boundary map composed with $\left(j_{0}\right)_{\#}$.

We can now finish the proof of our main results.

Proof of Theorem 23 and Theorem [30. Set $P=Z \cup_{\Psi} e^{n}$. By Lemma 35 (ii) the projections $p$ and $p^{*}$ extend to $X \stackrel{\hat{p}}{\leftarrow} P \stackrel{\hat{p}^{*}}{\rightarrow} X^{*}$. By a Mayer-Vietoris argument and Van Kampen theorem we get that the double-mapping-cylinder $X \times\{0\} \cup P \times$ $[0,1] \cup X^{*} \times\{1\}$ is a simply-connected homology sphere of dimension $n+1$. In other words we have a homotopy push-out

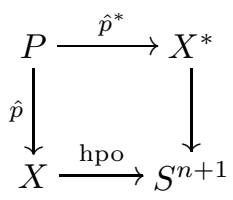

Moreover in Section 6 we will prove

Proposition 36. $P$ is a Poincaré duality space of dimension n.

Taking this proposition for granted, we continue the proof of Theorems 23 and 30] By Theorem 33 there exist compact submanifolds $N, N^{*}$ of codimension 0 in $S^{n+1}$ such that $N \cup N^{*}=S^{n+1}, N \cap N^{*}=\partial N=\partial N^{*}, N \simeq X, N^{*} \simeq X^{*}, \partial N \simeq P$. In other words we have the following push-out

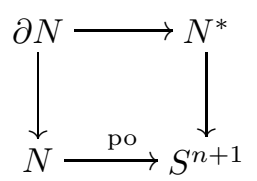

and the following homotopy commutative diagram, in which vertical arrows are homotopy equivalences:

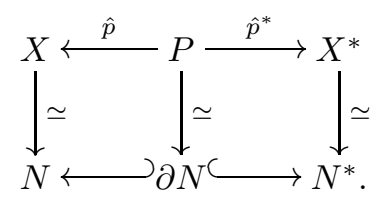

Since $X \simeq N$, we have proved that $X$ h-embeds in $S^{n+1}$. Moreover, $\partial N \simeq P$ as asserted in Theorem 30 and this homotopy boundary is tractable since the composite $N \simeq X \hookrightarrow Z \hookrightarrow P \simeq \partial N$ is a homotopy section of the inclusion $\partial N \hookrightarrow$ $N$. This proves Theorems 23 and 30 ,

\section{Proofs of Lemmas 34 and 35}

In this section we give the proofs of Lemmas 34 and 35 stated in the previous section. We use the notation of Sections 2.3 and 2.4 and of the previous section. 
Proof of Lemma 34 Recall from (77) before Lemma 21] the maps $\bar{w}_{k}: \Sigma B^{\wedge k} \wedge A^{*} \rightarrow$ $\Sigma B \vee \Sigma A^{*}(k \geq 0)$ defined inductively by $\bar{w}_{0}=\Sigma A^{*}: \Sigma A^{*} \hookrightarrow \Sigma B \vee \Sigma A^{*}$ and $\bar{w}_{k+1}=$ $\left[\Sigma B, \bar{w}_{k}\right]=\left(\Sigma B, \bar{w}_{k}\right) \bar{w}_{B, B^{\wedge k} \wedge A^{*}}$. In order to establish the equality $\left[f, \Sigma A^{*}\right] \circ \alpha=$ $[\Sigma B, \phi] \circ \beta$ in $\pi_{n-2}\left(Z_{0}\right)$, it suffices to prove that the following diagram is homotopy commutative:

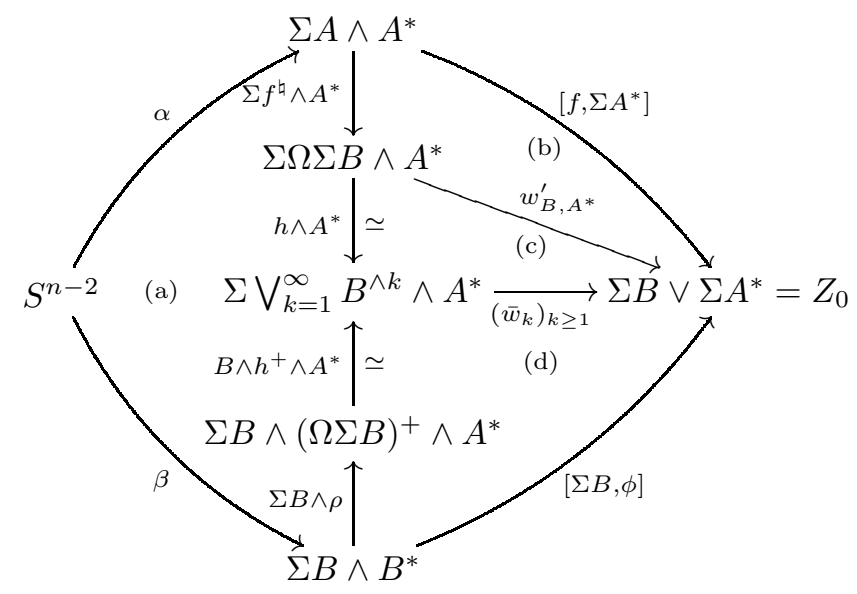

The subdiagram (19a) is exactly the diagram (11) of Section 4. It is therefore homotopy commutative by the main hypothesis of Theorem 23 The triangle (19p) commutes up to homotopy by Lemma 18 (ii), and triangle (19k) by Lemma 21 It remains to proving that $(19 \mathrm{~d})$ is homotopy commutative, which is equivalent to proving the following equation between homotopy classes:

$$
[\Sigma B, \phi]=\left(\overline{w_{k}}\right)_{k \geq 1} \circ\left(B \wedge h^{+} \wedge A^{*}\right) \circ(\Sigma B \wedge \rho) .
$$

Recall from (13) that $\phi=\phi^{\prime}+\phi^{\prime \prime}$, where $\phi^{\prime}=w_{B, A^{*}}^{\prime} \circ \Sigma \rho^{\prime}$ and $\phi^{\prime \prime}=\Sigma A^{*} \circ \Sigma \rho^{\prime \prime}$. First we show that

$$
\left[\Sigma B, \phi^{\prime}\right]=\left(\bar{w}_{l}\right)_{l \geq 2} \circ\left(B \wedge h \wedge A^{*}\right) \circ\left(\Sigma B \wedge \rho^{\prime}\right) .
$$

The latter equation is equivalent to the commutativity up to homotopy of the outer square of the following diagram:

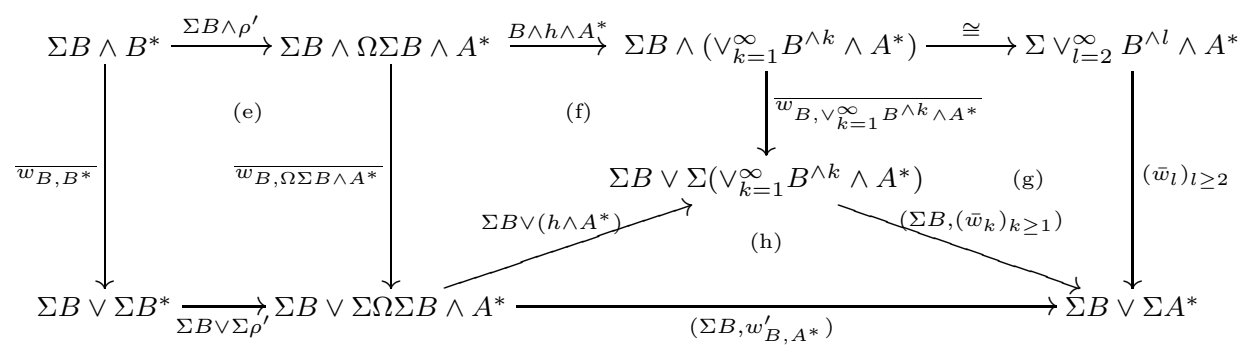

Subdiagrams (22) and (22f) are commutative by Lemma 18 (iii) (note that $h \wedge A^{*}$ is a suspension by our hypothesis on $\left.A^{*}\right),(22 \mathrm{~g})$ is commutative by the definition of $\bar{w}_{k+1}=\left[\Sigma B, \bar{w}_{k}\right]$, and (22) $\left.\mathrm{h}\right)$ commutes by Lemma 21. Thus diagram (22) is commutative, which proves equation (21).

On the other hand, we have

$$
\left[\Sigma B, \phi^{\prime \prime}\right]=\bar{w}_{1} \circ\left(\Sigma B \wedge \rho^{\prime \prime}\right)
$$


as a consequence of the commutativity up to homotopy of the diagram

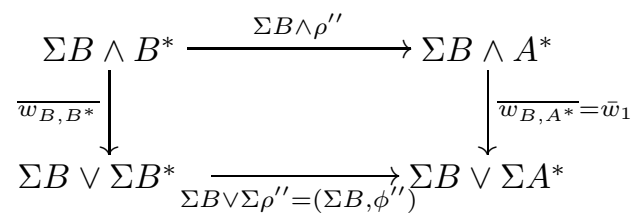

which follows by Lemma 18 (iii).

Combining equations (21) and (23), we now prove equation (20). Since $B^{*}$ is a suspension by the hypothesis of our main theorem, the Whitehead product $\left[\Sigma B, \phi^{\prime}+\phi^{\prime \prime}\right]$ is linear in the second variable (see [6, $(0.4)$, p. 29] or [3, remark after Korollar of Satz 2.11].) Therefore

$$
\begin{aligned}
{[\Sigma B, \phi] } & =\left[\Sigma B, \phi^{\prime}\right]+\left[\Sigma B, \phi^{\prime \prime}\right] \\
& =\left(\bar{w}_{l}\right)_{l \geq 2}\left(B \wedge h \wedge A^{*}\right)\left(\Sigma B \wedge \rho^{\prime}\right)+\bar{w}_{1}\left(\Sigma B \wedge \rho^{\prime \prime}\right) \\
& =\left(\bar{w}_{k}\right)_{k \geq 1}\left(B \wedge h^{+} \wedge A^{*}\right)\left(\Sigma B \wedge\left(\rho^{\prime}+\rho^{\prime \prime}\right)\right) \\
& =\left(\bar{w}_{k}\right)_{k \geq 1}\left(B \wedge h^{+} \wedge A^{*}\right)(\Sigma B \wedge \rho)
\end{aligned}
$$

because $\Sigma \rho=\Sigma \rho^{\prime}+\Sigma \rho^{\prime \prime}$ (see equation (12)). This establishes equation (20), which implies that the square (19d) is commutative. This finishes the proof of Lemma 34

Before proving Lemma 35 we need the following:

Lemma 37. Using the projection maps $q, q^{*}, p$ and $p^{*}$ defined at 14] and (15), we have:

(i) $\left(q, p_{0}\right) \Omega_{+}=0$ in $\pi_{n-1}\left(X \vee \Sigma^{2} B^{*}, \Sigma B\right)$ and $\left(q^{*}, p_{0}^{*}\right) \Omega_{-}=0$ in $\pi_{n-1}\left(X^{*} \vee\right.$ $\left.\Sigma^{2} A, \Sigma A^{*}\right)$;

(ii) $\left(p, p_{0}\right)\left(\Omega_{+}\right)=\left(p, p_{0}\right)\left(\Omega_{-}\right)=\left(p, p_{0}\right)(\Omega)=0$ in $\pi_{n-1}(X, \Sigma B)$; and

(iii) $\left(p^{*}, p_{0}^{*}\right)\left(\Omega_{+}\right)=\left(p^{*}, p_{0}^{*}\right)\left(\Omega_{-}\right)=\left(p^{*}, p_{0}^{*}\right)(\Omega)=0$ in $\pi_{n-1}\left(X^{*}, \Sigma A^{*}\right)$.

Proof of Lemma 37 For (i), consider the following diagram:

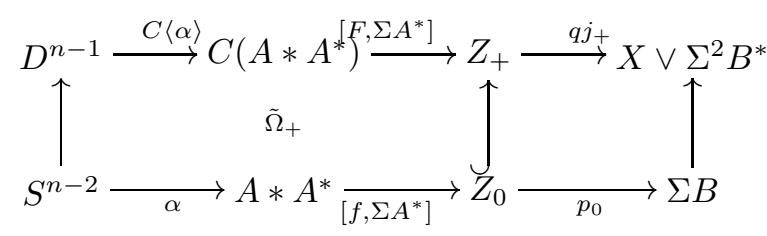

We compute

$$
\begin{aligned}
\left(q, p_{0}\right)\left(\Omega_{+}\right) & =\left(q j_{+}\left[F, \Sigma A^{*}\right] C\langle\alpha\rangle, p_{0}\left[f, \Sigma A^{*}\right] \alpha\right) \\
& =\left(\left[q j_{+} F,\left.p_{0}\right|_{\Sigma A^{*}}\right] C\langle\alpha\rangle,\left[p_{0} f, p_{0} \mid \Sigma A^{*}\right] \alpha\right) \\
& =\left[\left(q j_{+} F,\left.p_{0}\right|_{\Sigma A^{*}}\right),\left.p_{0}\right|_{\Sigma A^{*}}\right](C\langle\alpha\rangle, \alpha)
\end{aligned}
$$

and this relative homotopy class is trivial because the second factor of the relative 
GWP is $\left.p_{0}\right|_{\Sigma A^{*}}$, which is trivial in $\left[\Sigma A^{*}, \Sigma B\right]$. The vanishing of $\left(q^{*}, p_{0}^{*}\right) \Omega_{-}$is proved similarly.

For (ii), the equality $\left(p, p_{0}\right)\left(\Omega_{+}\right)=0$ follows from (i). On the other hand, we have $\left(p, p_{0}\right)\left(\Omega_{-}\right)=\left(p j_{-}, p_{0}\right)\left(\tilde{\Omega}_{-}\right)=0$ in $\pi_{n-1}(X, \Sigma B)$ because $p j_{-}: Z_{-} \rightarrow X$ factors through $\Sigma B$. From these two equalities we get that $\left(p, p_{0}\right)(\Omega)=\left(p, p_{0}\right)\left(\Omega_{+}\right)-$ $\left(p, p_{0}\right)\left(\Omega_{-}\right)=0$.

The point (iii) is proved as (ii), using the equality $\left(q^{*}, p_{0}^{*}\right) \Omega_{-}=0$ of (i).

Proof of Lemma 35. We have the following diagram, in which each vertical line is exact by the long exact sequence of homotopy groups of a pair:

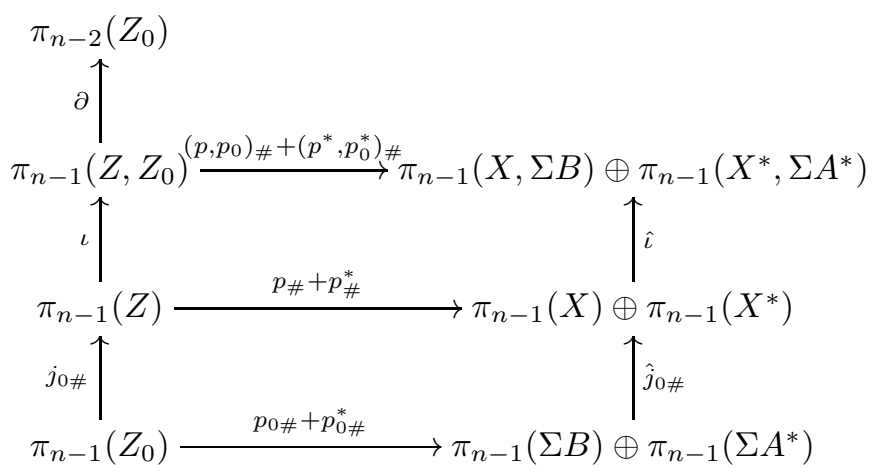

Since $\partial \Omega=\partial \Omega_{+}-\partial \Omega_{-}=\left[f, \Sigma A^{*}\right] \circ \alpha-[\Sigma B, \phi] \circ \beta=0$, there exists $\Theta \in \pi_{n-1}(Z)$ such that $\iota(\Theta)=\Omega$. By Lemma 37 we have $\left(p, p_{0}\right)_{\#}(\Omega)=0$ and $\left(p^{*}, p_{0}^{*}\right)_{\#}(\Omega)=0$; thus $p_{\#}(\Theta)+p_{\#}^{*}(\Theta) \in \operatorname{ker} \hat{\imath}=\operatorname{im} \hat{j}_{0 \#}$. Take $\left(\xi, \xi^{*}\right) \in \pi_{n-1}(\Sigma B) \oplus \pi_{n-1}\left(\Sigma A^{*}\right)$ such that $\hat{j}_{0 \#}\left(\xi, \xi^{*}\right)=\left(p_{\#}(\Theta), p_{\#}^{*}(\Theta)\right)$. The homomorphism $p_{0 \#}+p_{0 \#}^{*}$ is a split epimorphism because the map $\Omega\left(\Sigma B \vee \Sigma A^{*}\right) \stackrel{\Omega\left(p_{0}, p_{0}^{*}\right)}{\rightarrow} \Omega\left(\Sigma B \times \Sigma A^{*}\right)$ admits a homotopy section. Thus there exists $\zeta \in \pi_{n-1}\left(Z_{0}\right)$ such that $p_{0 \#}(\zeta)+p_{0 \#}^{*}(\zeta)=\left(\xi, \xi^{*}\right)$. Set $\Psi=\Theta-j_{0 \#}(\zeta) \in \pi_{n-1}(Z)$. Then $\iota(\Psi)=\Omega$ and $p_{\#}(\Psi)=p_{\#}^{*}(\Psi)=0$. Statement (iii) of Lemma 35 is proved with the same kind of diagram chasing.

\section{Proof that $P$ is a Poincaré duality space}

This section is devoted to the proof of Proposition 36 Let $f: \Sigma A \rightarrow \Sigma B$, $\alpha: S^{n-2} \rightarrow \Sigma A \wedge A^{*}$ and $\beta: S^{n-2} \rightarrow \Sigma B \wedge B^{*}$ be as in the statement of Theorem 23 We consider the same spaces and maps as in Section 4 In particular, recall the spaces

$$
\begin{aligned}
X & =\Sigma B \cup_{f} C \Sigma A, \\
X^{*} & =\Sigma A^{*} \cup_{f^{*}} C \Sigma B^{*}, \\
Z_{0} & =\Sigma B \vee \Sigma A^{*}, \\
Z & =\Sigma B \vee \Sigma A^{*} \cup_{f} C \Sigma A \cup_{\phi} C \Sigma B^{*}, \\
P & =Z \cup_{\Psi} e^{n},
\end{aligned}
$$


and the maps

$$
\begin{aligned}
p & : \quad Z \rightarrow X, \\
p^{*} & : \quad Z \rightarrow X^{*}, \\
\hat{p} & : \quad P \rightarrow X, \\
\hat{p}^{*} & : \quad P \rightarrow X^{*}, \\
p_{0} & : Z_{0} \rightarrow \Sigma B, \\
p_{0}^{*} & : \quad Z_{0} \rightarrow \Sigma A^{*}, \\
q & : \quad Z \rightarrow Z / \Sigma A^{*} \stackrel{g \simeq}{\rightarrow} X \vee \Sigma^{2} B^{*}, \\
q^{*} & : \quad Z \rightarrow Z / \Sigma B \stackrel{g^{*}=}{\rightarrow} X^{*} \vee \Sigma^{2} A .
\end{aligned}
$$

We have also the homotopy classes

$$
\begin{aligned}
& \Omega=\Omega_{+}-\Omega_{-} \in \pi_{n-1}\left(Z, Z_{0}\right), \\
& \Psi \in \pi_{n-1}(Z), \text { which is a lift of } \Omega .
\end{aligned}
$$

In all this section, we fix a field $\mathbb{K}$ and denote by $H^{*}$ the cohomology with coefficients in $\mathbb{K}$. We exhibit now a suitable basis of $H^{*}(P)$. Since $\alpha$ is an $(n-$ 2 - coduality, there exist homogeneous bases $\left\{a_{i}\right\}_{1 \leq i \leq p}$ of $\tilde{H}^{*}(A)$ and $\left\{a_{i}^{*}\right\}_{1 \leq i \leq p}$ of $\tilde{H}^{*}\left(A^{*}\right)$ such that $H^{*}(\alpha)\left(\sigma\left(a_{i} \wedge a_{j}^{*}\right)\right)=\delta_{i j}\left[S^{n-2}\right]$, where $\delta_{i j}$ is the Kronecker symbol and $\left[S^{n-2}\right] \in H^{n-2}\left(S^{n-2)}\right.$ is a fundamental class. Similarly we have bases $\left\{b_{j}\right\}_{1 \leq j \leq q}$ of $\tilde{H}^{*}(B)$ and $\left\{b_{j}^{*}\right\}_{1 \leq j \leq q}$ of $\tilde{H}^{*}\left(B^{*}\right)$ such that $H^{*}(\beta)\left(\sigma\left(b_{i} \wedge b_{j}^{*}\right)\right)=\delta_{i j}\left[S^{n-2}\right]$.

By the hypothesis of our main theorem, $\tilde{H}^{*}(f)=0$. On the other hand, $f^{*}=$ $p_{0}^{*} \phi=\Sigma \rho^{\prime \prime}$ is a representative of the S-dual of $f$ (see Remark 3 after the statement of Theorem 23), and this implies that $\tilde{H}^{*}\left(f^{*}\right)=0$ as well. Therefore we have two short exact sequences

$$
\begin{aligned}
& 0 \rightarrow \tilde{H}^{*}\left(\Sigma^{2} A\right) \rightarrow \tilde{H}^{*}(X) \rightarrow \tilde{H}^{*}(\Sigma B) \rightarrow 0, \\
& 0 \rightarrow \tilde{H}^{*}\left(\Sigma^{2} B^{*}\right) \rightarrow \tilde{H}^{*}\left(X^{*}\right) \rightarrow \tilde{H}^{*}\left(\Sigma A^{*}\right) \rightarrow 0 .
\end{aligned}
$$

Using these exact sequences, denote by $\alpha_{i}$ the image of $\sigma^{2} a_{i}$ in $\tilde{H}^{*}(X)$ and by $\beta_{j}^{*}$ the image of $\sigma^{2} b_{j}^{*}$ in $\tilde{H}^{*}\left(X^{*}\right)$. Let $\beta_{j}$ be elements in $\tilde{H}^{*}(X)$ that project onto $\sigma b_{j}$, and let $\alpha_{i}^{*}$ be elements in $\tilde{H}^{*}\left(X^{*}\right)$ that project onto $\sigma a_{i}^{*}$. Then the family $\left\{\alpha_{i}, \beta_{j}\right\}$ is a basis of $\tilde{H}^{*}(X)$, and $\left\{\alpha_{i}^{*}, \beta_{j}^{*}\right\}$ is a basis of $\tilde{H}^{*}\left(X^{*}\right)$.

We denote also by $\alpha_{i}, \beta_{j}, \alpha_{i}^{*}, \beta_{j}^{*}$ the images of these cohomology classes in $H^{*}(P)$ (resp. in $\left.H^{*}(Z)\right)$ under the morphisms $H^{*}(\hat{p})$ and $H^{*}\left(\hat{p}^{*}\right)$ (resp. $H^{*}(p)$ and $H^{*}\left(p^{*}\right)$.) Let $[P] \in H^{n}(P)$ be the image of the fundamental class $\left[S^{n}\right] \in H^{n}\left(S^{n}\right)$ by the pinching map $P \rightarrow S^{n}$. Then

$$
\left\{1, \beta_{j}, \alpha_{i}^{*}, \alpha_{i}, \beta_{j}^{*},[P]\right\}
$$

is a basis of $H^{*}(P)$.

Let $\mu \in H_{n}(P)$ be the dual class of $[P] \in H^{n}(P)$. In order to prove that $H^{*}(P)$ is a Poincaré duality algebra we will establish formulas like $\left\langle\alpha_{i} \cup \alpha_{j}^{*}, \mu\right\rangle=\delta_{i j}$, $\left\langle\beta_{i} \cup \beta_{j}^{*}, \mu\right\rangle=\delta_{i j}$, etc. (see Lemma 41 below for the precise statement). With this aim we introduce maps $\Lambda_{ \pm}$and $\overline{j_{ \pm}}$.

Consider the homotopy equivalences established in Lemma 20

$$
\begin{array}{ll}
\theta_{+}: & \Sigma\left(A * A^{*}\right) \stackrel{\simeq}{\rightrightarrows}(\Sigma A) * A^{*}, \\
\theta_{-}: & \Sigma\left(B * B^{*}\right) \stackrel{\simeq}{\rightrightarrows} B *\left(\Sigma B^{*}\right) .
\end{array}
$$


Define the composites

$$
\begin{aligned}
& \Lambda_{+}: S^{n-1} \stackrel{\Sigma \alpha}{\longrightarrow} \Sigma\left(A * A^{*}\right) \stackrel{\theta_{+}}{\simeq}(\Sigma A) * A^{*} \stackrel{\overline{w_{\Sigma A, A}}}{\longrightarrow} \Sigma A^{*} \vee \Sigma^{2} A, \\
& \Lambda_{-}: S^{n-1} \stackrel{\Sigma \beta}{\longrightarrow} \Sigma\left(B * B^{*}\right) \stackrel{\theta_{-}}{\longrightarrow} B *\left(\Sigma B^{*}\right) \stackrel{\bar{w}_{B, \Sigma B^{*}}}{\longrightarrow} \Sigma B \vee \Sigma^{2} B^{*}
\end{aligned}
$$

and consider the inclusions

$$
\begin{array}{ll}
\overline{j_{+}}: & \quad \Sigma A^{*} \vee \Sigma^{2} A \hookrightarrow X^{*} \vee \Sigma^{2} A, \\
\overline{j_{-}}: & \quad \Sigma B \vee \Sigma^{2} B^{*} \hookrightarrow X \vee \Sigma^{2} B^{*} .
\end{array}
$$

The key idea in proving that $P$ is a Poincare duality space is to study the following diagram of cofibration sequences:

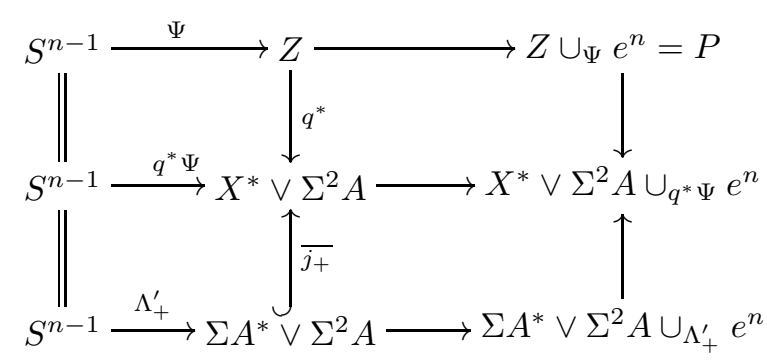

where the map $\Lambda_{+}^{\prime}$, still to be defined, will be related to $\Lambda_{+}$. We will prove that the cohomology of the space $\left(\Sigma A^{*} \vee \Sigma^{2} A\right) \cup_{\Lambda_{+}^{\prime}} e^{n}$ satisfies the formula $\sigma a_{i}^{*} \cup \sigma^{2} a_{j}=\delta_{i j}\left[e^{n}\right]$ (Lemma 40). Using diagram (24), we will deduce that $\alpha_{i}^{*} \cup \alpha_{j}=\delta_{i j}[P]$ in $H^{n}(P)$. Similarly we have $\beta_{i}^{*} \cup \beta_{j}=\delta_{i j}[P]$ in $H^{n}(P)$. From this we will deduce that $P$ satisfies Poincaré duality.

To develop this line of proof we need to establish a few lemmas. The next two lemmas will permit us to construct the map $\Lambda_{+}^{\prime}$ related to $\Lambda_{+}$and making diagram (24) commutative.

Lemma 38. Consider the following morphisms of homotopy groups:

$$
\begin{aligned}
& \pi_{n-1}\left(\Sigma A^{*} \vee \Sigma^{2} A\right) \stackrel{\left(\overline{j_{+}}\right) \#}{\longrightarrow} \pi_{n-1}\left(X^{*} \vee \Sigma^{2} A\right) \stackrel{\iota}{\longrightarrow} \pi_{n-1}\left(X^{*} \vee \Sigma^{2} A, \Sigma A^{*}\right), \\
& \pi_{n-1}\left(Z, Z_{0}\right) \stackrel{\left(q^{*}, p_{0}^{*}\right) \#}{\longrightarrow} \pi_{n-1}\left(X^{*} \vee \Sigma^{2} A, \Sigma A^{*}\right)
\end{aligned}
$$

and the homotopy classes $\Lambda_{+} \in \pi_{n-1}\left(\Sigma A^{*} \vee \Sigma^{2} A\right)$ and $\Omega \in \pi_{n-1}\left(Z, Z_{0}\right)$. Then

$$
\iota\left(\overline{j_{+}}\left(\Lambda_{+}\right)\right)=\left(q^{*}, p_{0}^{*}\right)_{\#}(\Omega) \in \pi_{n-1}\left(X^{*} \vee \Sigma^{2} A, \Sigma A^{*}\right) .
$$

Similarly,

$$
\iota\left(\overline{j_{-}}\left(\Lambda_{-}\right)\right)=\left(q, p_{0}\right)_{\#}(\Omega) \in \pi_{n-1}\left(X \vee \Sigma^{2} B^{*}, \Sigma B\right) .
$$

Proof. In $\pi_{n-1}\left(X^{*} \vee \Sigma^{2} A, \Sigma A^{*}\right)$ we have $\left(q^{*}, p_{0}^{*}\right) \Omega=\left(q^{*}, p_{0}^{*}\right)\left(\Omega_{+}-\Omega_{-}\right)=\left(q^{*}, p_{0}^{*}\right) \Omega_{+}$ since by Lemma $37(\mathrm{i})$ we have $\left(q^{*}, p_{0}^{*}\right) \Omega_{-}=0$. From (14) we see that the projection map

$$
q_{+}^{*}: Z \stackrel{\mathrm{pr}}{\rightarrow} Z_{+} / \Sigma B \stackrel{g_{+}^{*}=}{\longrightarrow} \Sigma A^{*} \vee \Sigma^{2} A
$$


makes commutative the following diagram:

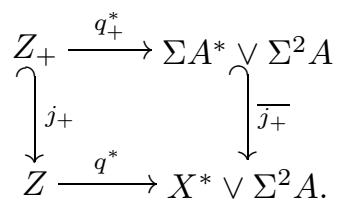

Recall from (16) that $\Omega_{+} \in \pi_{n-1}\left(Z, Z_{0}\right)$ is the image under $j_{+}$of the relative homotopy class $\tilde{\Omega}_{+} \in \pi_{n-1}\left(Z_{+}, Z_{0}\right)$ defined as

$$
\begin{aligned}
\tilde{\Omega}_{+} & =\left(\left[F, \Sigma A^{*}\right] \circ C\langle\alpha\rangle,\left[f, \Sigma A^{*}\right] \circ \alpha\right) \\
& =\left(\left(F, \Sigma A^{*}\right) \circ \overline{W_{A, A^{*}}} \circ C\langle\alpha\rangle,\left(f, \Sigma A^{*}\right) \circ \overline{w_{A, A^{*}}} \circ \alpha\right) .
\end{aligned}
$$

Since $q^{*} j_{+}=\overline{j_{+}} q_{+}^{*}$, the class $\left(q^{*}, p_{0}^{*}\right) \Omega_{+}=\left(q^{*}, p_{0}^{*}\right) j_{+} \tilde{\Omega}_{+}$can be represented by the following diagram, where $\left(\overline{W_{A, A^{*}}}, \overline{w_{A, A^{*}}}\right)$ is the universal relative generalized Whitehead product defined in Section 2.3 and $\xi$ and $\Xi$ are the canonical projections shrinking $\Sigma A$ to the base point:

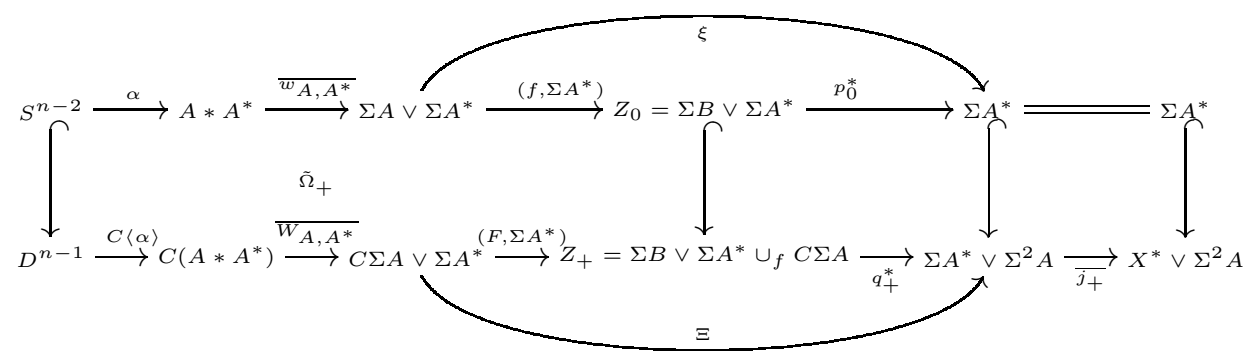

Therefore we have equalities between the relative homotopy classes

$$
\left(q^{*}, p_{0}^{*}\right) \Omega=\left(q^{*}, p_{0}^{*}\right) \Omega_{+}=\overline{j_{+}} \circ(\Xi, \xi) \circ\left(\overline{W_{A, A^{*}}}, \overline{w_{A, A^{*}}}\right) \circ(C\langle\alpha\rangle, \alpha) .
$$

On the other hand,

$$
\overline{j_{+}} \Lambda_{+}=\overline{j_{+}} \circ \overline{w_{\Sigma A, A^{*}}} \circ \theta_{+} \circ \Sigma \alpha .
$$

From Lemma 20(i) we know that $\overline{w_{\Sigma A, A^{*}}} \circ \theta_{+}$is a lifting of $(\Xi, \xi)\left(\overline{W_{A, A^{*}}}, \overline{w_{A, A^{*}}}\right)$ along $\Gamma$, and we conclude that $\overline{j_{+}} \Lambda_{+}$is a lifting of the class $\left(q^{*}, p_{0}^{*}\right) \Omega$ along $\iota$.

The proof of the formula $\iota\left(\overline{j_{-}}\left(\Lambda_{-}\right)\right)=\left(q, p_{0}\right)_{\#}(\Omega)$ is similar.

Lemma 39. There exists $\epsilon_{+} \in \pi_{n-1}\left(\Sigma A^{*}\right)$ such that $\overline{j_{+}}\left(\Lambda_{+}+\epsilon_{+}\right)=q_{\#}^{*}(\Psi) \in$ $\pi_{n-1}\left(X^{*} \vee \Sigma^{2} A\right)$.

Similarly, there exists $\epsilon_{-} \in \pi_{n-1}(\Sigma B)$ such that $\overline{j_{-\#}}\left(\Lambda_{-}+\epsilon_{-}\right)=q_{\#}(\Psi) \in$ $\pi_{n-1}\left(X \vee \Sigma^{2} B^{*}\right)$.

Proof. We want to prove that there exists a map $\epsilon_{+}: S^{n-1} \rightarrow \Sigma A^{*}$ making the following diagram homotopy commutative:

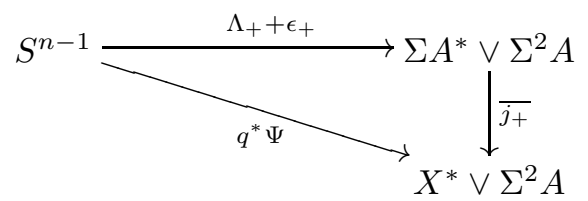


Consider the following commutative diagram:

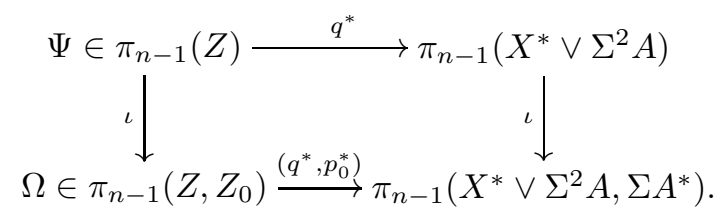

By Lemma 35(i) and the last diagram we see that $q^{*} \Psi$ is a lifting in $\pi_{n-1}\left(X^{*} \vee \Sigma^{2} A\right)$ of the class $\left(q^{*}, p_{0}^{*}\right) \Omega \in \pi_{n-1}\left(X^{*} \vee \Sigma^{2} A, \Sigma A^{*}\right)$ along $\iota$. By Lemma 38 we know that $\overline{j_{+}} \Lambda_{+} \in \pi_{n-1}\left(X^{*} \vee \Sigma^{2} A\right)$ is another lifting of $\left(q^{*}, p_{0}^{*}\right) \Omega$ along $\iota$. From the diagram of exact sequences

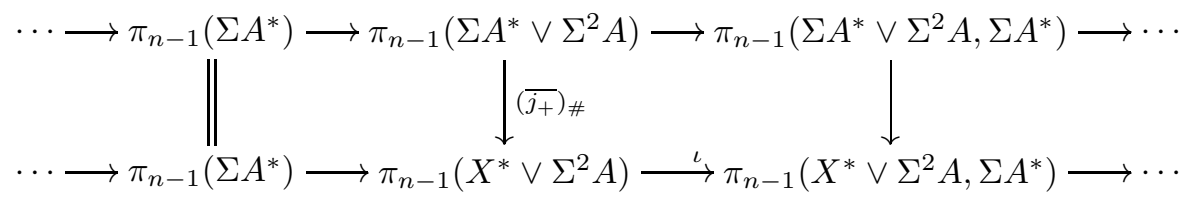

we deduce that the classes $\overline{j_{+}} \Lambda_{+}$and $q^{*} \Psi$ differ by some class $\epsilon_{+} \in \pi_{n-1}\left(\Sigma A^{*}\right)$. This homotopy class $\epsilon_{+}$makes diagram (25) homotopy commutative.

The proof of the existence of $\epsilon_{-}$is analogous. Lemma 39 is proved.

Set

$$
\begin{aligned}
& \Lambda_{+}^{\prime}:=\Lambda_{+}+\epsilon_{+} \in \pi_{n-1}\left(\Sigma A^{*} \vee \Sigma^{2} A\right), \\
& \Lambda_{-}^{\prime}:=\Lambda_{-}+\epsilon_{-} \in \pi_{n-1}\left(\Sigma B \vee \Sigma^{2} B^{*}\right),
\end{aligned}
$$

where $\epsilon_{+}$and $\epsilon_{-}$are the homotopy classes constructed in Lemma 39.

Lemma 40. In the cohomology of $\left(\Sigma A^{*} \vee \Sigma^{2} A\right) \cup_{\Lambda_{+}^{\prime}} e^{n}$ we have

$$
\sigma a_{i}^{*} \cup \sigma^{2} a_{j}= \pm \delta_{i j}\left[e^{n}\right]
$$

where $\left[e^{n}\right] \in H^{n}\left(\left(\Sigma A^{*} \vee \Sigma^{2} A\right) \cup_{\Lambda_{+}^{\prime}} e^{n}\right)$ is the pullback of the cohomology class $\left[S^{n}\right]$ under the pinching map $\left(\Sigma A^{*} \vee \Sigma^{2} A\right) \cup_{\Lambda_{+}^{\prime}} e^{n} \rightarrow S^{n}$.

Similarly, $\sigma b_{i} \cup \sigma^{2} b_{j}^{*}= \pm \delta_{i j}\left[e^{n}\right]$ in the cohomology of $\left(\Sigma B \vee \Sigma^{2} B^{*}\right) \cup_{\Lambda_{-}^{\prime}} e^{n}$.

Proof. By formula (ii) of Lemma 20 and Proposition [16 (0), $\theta_{+} \circ \Sigma \alpha$ is a coduality between $A^{*}$ and $\Sigma A$. It follows from Proposition 19 that the equations $\sigma a_{i}^{*} \cup \sigma^{2} a_{j}=$ $\pm \delta_{i j}\left[e^{n}\right]$ hold in $H^{*}\left(\Sigma A^{*} \vee \Sigma^{2} A \cup_{\Lambda_{+}} e^{n}\right)$. As $\epsilon_{+} \in \pi_{n-1}\left(\Sigma A^{*}\right)$, we have $\sigma a_{i}^{*} \cup \sigma^{2} a_{j}=0$ in $H^{*}\left(\Sigma A^{*} \vee \Sigma^{2} A \cup_{\epsilon_{+}} e^{n}\right)$. From this fact together with Theorem 4.1 of [21], we deduce that the equations $\sigma a_{i}^{*} \cup \sigma^{2} a_{j}= \pm \delta_{i j}\left[e^{n}\right]$ hold in $H^{*}\left(\Sigma A^{*} \vee \Sigma^{2} A \cup_{\Lambda_{+}^{\prime}} e^{n}\right)$. The proof for $\left(\Sigma B \vee \Sigma^{2} B^{*}\right) \cup_{\Lambda_{-}^{\prime}} e^{n}$ is analogous.

Recall that $\mu \in H_{n}(P ; \mathbb{K})$ is the homology class such that $\langle[P], \mu\rangle=1$, where $\langle.,$.$\rangle is the pairing between cohomology and homology. The Poincaré duality of P$ will be essentially a consequence of the following equations. 
Lemma 41. The cohomology algebra $H^{*}(P)$ satisfies the following equations:

$$
\begin{aligned}
& \left\langle\alpha_{i} \cup \alpha_{j}, \mu\right\rangle=0, \\
& \left\langle\beta_{i}^{*} \cup \beta_{j}^{*}, \mu\right\rangle=0, \\
& \left\langle\alpha_{i}^{*} \cup \beta_{j}^{*}, \mu\right\rangle=0, \\
& \left\langle\alpha_{i} \cup \beta_{j}^{*}, \mu\right\rangle=0, \\
& \left\langle\alpha_{i} \cup \alpha_{j}^{*}, \mu\right\rangle= \pm \delta_{i j}, \\
& \left\langle\beta_{i} \cup \beta_{j}^{*}, \mu\right\rangle= \pm \delta_{i j} .
\end{aligned}
$$

Proof. Equations (26), 27) and (28) come from the fact that the image of $\mu$ by the projections $P \stackrel{\hat{p}}{\rightarrow} X$ and $P \stackrel{\hat{p}^{*}}{\rightarrow} X^{*}$ is zero.

For Equation (29) consider the projection $r: Z \rightarrow Z / Z_{0}=\Sigma^{2} A \vee \Sigma^{2} B^{*}$ and the following diagram:

$$
\begin{array}{clcc}
\Omega \in & \pi_{n-1}\left(Z, Z_{0}\right) & \stackrel{r_{\#}}{\rightarrow} & \pi_{n-1}\left(\Sigma^{2} A \vee \Sigma^{2} B^{*}, *\right) \\
\uparrow & \uparrow \iota & & \uparrow= \\
\Psi \in & \pi_{n-1}(Z) & \stackrel{r_{\#}}{\rightarrow} & \pi_{n-1}\left(\Sigma^{2} A \vee \Sigma^{2} B^{*}\right)
\end{array}
$$

Note that $Z_{+} / Z_{0}=\Sigma^{2} A$ and $Z_{-} / Z_{0}=\Sigma^{2} B^{*}$. Since $\Omega=\Omega_{+}-\Omega_{-}$and since $\Omega_{ \pm} \in$ $\pi_{n-1}\left(Z, Z_{0}\right)$ lifts to $\tilde{\Omega}_{ \pm} \in \pi_{n-1}\left(Z_{ \pm}, Z_{0}\right)$, there exist elements $\xi^{\prime} \in \pi_{n-1}\left(\Sigma^{2} A, *\right)$ and $\xi^{\prime \prime} \in \pi_{n-1}\left(\Sigma^{2} B^{*}, *\right)$ such that $r_{\#}(\Omega)=\xi^{\prime}-\xi^{\prime \prime}$. Thus $r_{\#}(\Psi)=\xi^{\prime}-\xi^{\prime \prime}$. We have the following diagram of cofibration sequences:

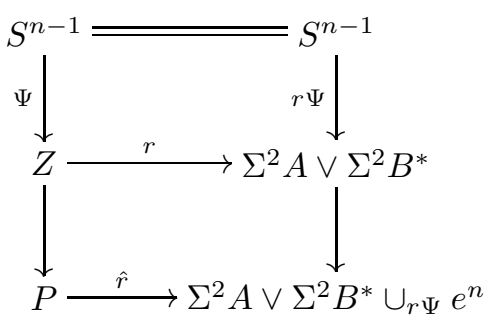

Since $r_{\#} \Psi=\xi^{\prime}-\xi^{\prime \prime} \in \pi_{n-1}\left(\Sigma^{2} A\right)+\pi_{n-1}\left(\Sigma^{2} B^{*}\right)$, the cup products $\sigma^{2} a_{i} \cup \sigma^{2} b_{j}^{*}$ are zero in $H^{*}\left(\Sigma^{2} A \vee \Sigma^{2} B^{*} \cup_{r} \Psi e^{n}\right)$. Since $H^{*}(\hat{r})\left(\sigma^{2} a_{i}\right)=\alpha_{i}$ and $H^{*}(\hat{r})\left(\sigma^{2} b_{j}^{*}\right)=\beta_{j}^{*}$, we get that $\left\langle\alpha_{i} \cup \beta_{j}^{*}, \mu\right\rangle=0$ in $H^{*}(P)$ because $H^{n}(\hat{r})$ is an isomorphism. This proves equation (29).

Equation (30). Consider the following diagram of cofibration sequences, in which the left square is commutative by Lemma 39 and where $\hat{j_{+}}$is the induced map between the cofibres:

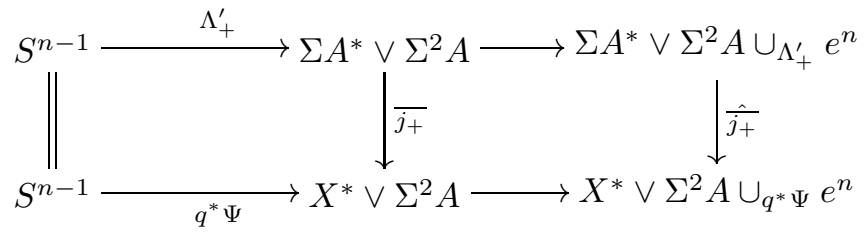

The image in $H^{*}\left(\Sigma A^{*} \vee \Sigma^{2} A\right)$ of the family $\left\{\sigma^{2} a_{i}, \alpha_{i}^{*}\right\} \subset H^{*}\left(X^{*} \vee \Sigma^{2} A \cup_{q^{*} \Psi} e^{n}\right)$ under $H^{*}\left(\hat{\overline{j_{+}}}\right)$is $\left\{\sigma^{2} a_{i}, \sigma a_{i}^{*}\right\}$. By Lemma 40 we have

$$
\sigma a_{i}^{*} \cup \sigma^{2} a_{j}= \pm \delta_{i j}\left[e^{m}\right]
$$


in the cohomology of $\Sigma A^{*} \vee \Sigma^{2} A \cup_{\Lambda_{+}^{\prime}} e^{n}$. Since the map $\hat{j_{+}}$induces an isomorphism in cohomology in degree $n$, we deduce that

$$
\alpha_{i}^{*} \cup \sigma^{2} a_{i}=\delta_{i j} \cdot\left[e^{n}\right] \text { in } H^{n}\left(X^{*} \vee \Sigma^{2} A \cup_{q^{*} \Psi} e^{n}\right) .
$$

Consider the following commutative diagram of cofibration sequences:

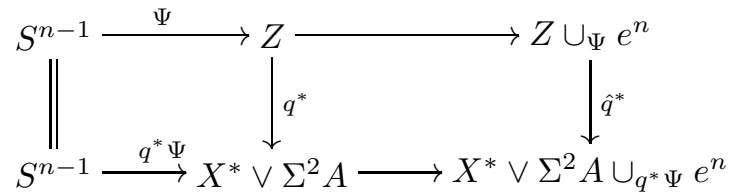

where $\hat{q}^{*}$ is the map between the cofibres induced by $q^{*}$. The image under $H^{*}\left(q^{*}\right)$ of the family $\left\{\sigma^{2} a_{i}, \alpha_{i}^{*}\right\} \subset H^{*}\left(X^{*} \vee \Sigma^{2} A\right)$ is $\left\{\alpha_{i}, \alpha_{i}^{*}\right\}$. Since $H^{n}\left(\hat{q}^{*}\right)$ is an isomorphism which sends $\left[e^{n}\right]$ on $[P]$, equation (32) implies that

$$
\alpha_{i}^{*} \cup \alpha_{j}= \pm \delta_{i j}[P] \text { in } H^{n}(P) .
$$

This establishes equation (30).

Equation (31) is proved similarly to equation (30) by considering the diagram

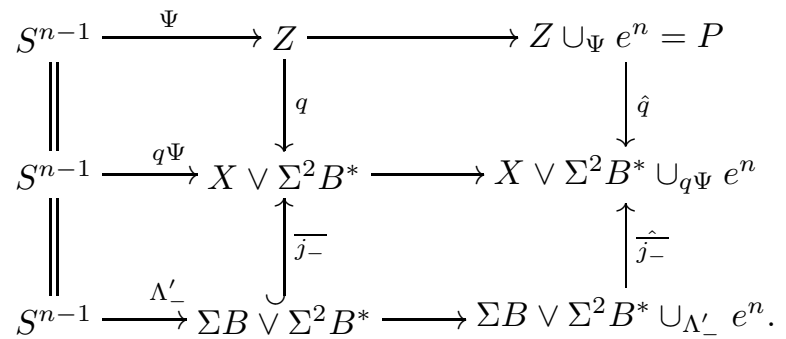

We can now complete the proof of the Poincaré duality of $P$.

Proof of Proposition 36. Consider the bilinear form

$$
\zeta: H^{*}(P) \otimes H^{*}(P) \rightarrow \mathbb{K}, \quad u \otimes v \mapsto\langle u \cup v, \mu\rangle .
$$

By Lemma 41 the matrix of this bilinear form in the basis

$$
\left\{1, \beta_{1}, \ldots, \beta_{q}, \alpha_{1}^{*}, \ldots, \alpha_{p}^{*}, \alpha_{1}, \ldots, \alpha_{p}, \beta_{1}^{*}, \ldots, \beta_{q}^{*},[P]\right\}
$$

of $H^{*}(P)$ has the form

$$
M=\left(\begin{array}{cccccc}
0 & 0 & 0 & 0 & 0 & 1 \\
0 & * & * & * & I_{q} & 0 \\
0 & * & * & I_{p} & 0 & 0 \\
0 & * & I_{p}^{\prime} & 0 & 0 & 0 \\
0 & I_{q}^{\prime} & 0 & 0 & 0 & 0 \\
1 & 0 & 0 & 0 & 0 & 0
\end{array}\right)
$$

where $I_{p}$ and $I_{p}^{\prime}$ (resp. $I_{q}$ and $I_{q}^{\prime}$ ) is a diagonal matrix of rank $p$ (resp. $q$ ) with only \pm 1 on the diagonal, $*$ is any matrix and 0 is the zero-matrix. The determinant of this matrix $M$ is \pm 1 , so the bilinear form $\zeta$ is non-degenerate, and thus the cohomology algebra $H^{*}(P ; \mathbb{K})$ satisfies Poincaré duality. Since this is true for any field $\mathbb{K}$, we have that $P$ is a Poincaré duality space by [9] Proposition I.2.1]. 


\section{EMBEDDINGS UP TO RATIONAL HOMOTOPY}

In this section we prove that there are no rational obstructions to h-embeddings of simply-connected two-cones in codimension 3. We suggest the following:

Conjecture 42. Given a simply-connected finite $C W$-complex $X$ of dimension $n$, there exist a $C W$-complex $X^{\prime}$ rationally homotopy equivalent to $X$ and a tractable $h$-embedding $X^{\prime} \stackrel{h}{\subset} S^{n+3}$.

In fact we are not even aware of any rational obstruction to h-embedding in codimension 2. We remark that by a theorem of Baues 5 there always exists a CW-complex $X^{\prime}$ having the rational homotopy type of $X$ and such that $\Sigma X^{\prime}$ is homotopy equivalent to a wedge of spheres; thus $\Sigma X^{\prime}$ h-embeds in $S^{n+2}$. But this does not imply the conjecture: we now give examples for arbitrarily large $i$ of CW-complexes $X$ such that for some $n, \Sigma X$ is homotopy equivalent to a wedge of spheres of dimension less than $n+1$ although $X \not{\not} S^{n+i+1}$.

Example 43. For any integer $r \geq 1$ there exists a stable homotopy class $\zeta \in \pi_{*}^{S}$ of odd order whose sphere of origin is higher than $S^{r}$, i.e. $\zeta \notin \operatorname{im}\left(\pi_{*+r}\left(S^{r}\right) \rightarrow \pi_{*}^{S}\right)$. Therefore for an arbitrarily large $i$ we can find an even integer $m \geq i+7$ and a homotopy class $g \in \pi_{n-2}\left(S^{2 m-2}\right)$ such that $2\{g\} \notin \operatorname{im}\left(\pi_{n-6}\left(S^{2 m-6}\right) \rightarrow \pi_{2 m-n}^{S}\right)$. Set $f=\left[S^{m}, S^{m}\right] \circ \Sigma g \in \pi_{n-1}\left(S^{m}\right)$. Then $\gamma^{2}(f)=\gamma^{2}\left(\left[S^{m}, S^{m}\right]\right) \circ \Sigma g=2 \Sigma g$. Therefore if $k<m-5$ there is no $\psi_{0} \in \pi_{n+k-1-m}\left(S^{m+k}\right)$ such that $\Sigma^{k-1} \gamma^{2}(f)=$ $\Sigma^{m-1} \psi_{0}$. Using condition (ii) of the paper of Cooke [11, p. 145, we get that $X=S^{m} \cup_{f} e^{n}$ does not h-embed in $S^{n+i+1}$. On the other hand, $\Sigma f=0$ and $\Sigma X \simeq S^{m+1} \vee S^{n+1}$.

We prove the above conjecture for two-cones, which was stated as Theorem 7 in the Introduction:

Proof of Theorem 7 . Let $X$ be the homotopy cofibre of some map $\Sigma A \stackrel{f}{\rightarrow} \Sigma B$ between finite suspensions. Set $n=\operatorname{dim} X+2$. Since suspensions are rationally equivalent to wedges of spheres, there exist finite wedges $U=\bigvee_{i=1}^{p} S^{a_{i}}$ and $V=\bigvee_{j=1}^{q} S^{b_{j}}$ and a map $g: \Sigma U \rightarrow \Sigma V$ whose homotopy cofibre $X^{\prime \prime}$ has the same rational homotopy type as $X$. Moreover we can choose $\Sigma U, \Sigma V$ and $g$ such that $\tilde{H}_{*}(g ; \mathbb{Z})=0$ and such that $\operatorname{dim} \Sigma U>\operatorname{dim} \Sigma V$, which implies that $n \geq \max \left(a_{i}, b_{j}\right)+4$.

For the sake of the proof we introduce the following definition of a linear map between wedges of spheres. A map $\ell: S^{m} \rightarrow S^{n}$ between two spheres is called linear if either $\ell$ is homotopy trivial or $m=n$, in which case $\ell$ is determined by its degree. A map $\ell=\left(\ell_{\alpha}\right)_{\alpha}: \bigvee_{\alpha} S^{m_{\alpha}} \rightarrow \bigvee_{\beta} S^{n_{\beta}}$ is called linear if each of the maps $\ell_{\alpha} \in\left[S^{m_{\alpha}}, \vee_{\beta} S^{n_{\beta}}\right]$ is a sum of linear maps between two spheres. Thus there is an obvious bijective correspondence

$$
\left\{\ell \in\left[\bigvee_{\alpha} S^{m_{\alpha}}, \bigvee_{\beta} S^{n_{\beta}}\right]: \ell \text { is linear }\right\} \longleftrightarrow \operatorname{hom}_{\mathbb{Z}}\left(\tilde{H}_{*}\left(\bigvee_{\alpha} S^{m_{\alpha}} ; \mathbb{Z}\right), \tilde{H}_{*}\left(\bigvee_{\beta} S^{n_{\beta}} ; \mathbb{Z}\right)\right)
$$

For $N \in \mathbb{Z}$ we commit the abuse of notation of denoting also by $N$ the map from $\Sigma U$ to itself of degree $N$. In other words, $N \in[\Sigma U, \Sigma U]$ is the linear map such that $\tilde{H}_{*}(N ; \mathbb{Z})$ is $N$ times the identity. 
Consider the composite

$$
\theta: \Sigma U \stackrel{\Sigma\left(g^{\natural}\right)}{\longrightarrow} \Sigma \Omega \Sigma V \stackrel{\simeq h}{\longrightarrow} \Sigma\left(\bigvee_{k=1}^{\infty} V^{\wedge k}\right),
$$

which is a map between wedges of spheres. There exists a unique linear map $\ell \in\left[\Sigma^{2} U, \Sigma^{2}\left(\bigvee_{k=1}^{\infty} V^{\wedge k}\right)\right]$ such that $H_{*}(\ell ; \mathbb{Z})=H_{*}(\Sigma \theta ; \mathbb{Z})$. By standard arguments in rational homotopy theory two suspensions of maps which agree in homology are rationally homotopic, and since $\ell$ is obviously a suspension we have $(\ell)_{\mathbb{Q}}=(\Sigma \theta)_{\mathbb{Q}}$. Therefore, since $\Sigma^{2} U$ is a finite wedge of spheres, there exists a non-zero integer $N$ such that $\Sigma \theta \circ \Sigma N=\ell \circ \Sigma N$.

Set $g^{\prime}=g \circ N$ and $\theta^{\prime}=h \circ \Sigma\left(g^{\prime}\right)^{\natural}$. Since the adjunction $-^{\natural}:[\Sigma U, Z] \rightarrow[U, \Omega Z]$ and the suspension $\Sigma:[\Sigma U, Z] \rightarrow\left[\Sigma^{2} U, \Sigma Z\right]$ are homomorphisms of groups, we have that $\Sigma\left(g^{\prime}\right)^{\natural}=\left(\Sigma g^{\natural}\right) \circ N$. Hence $\theta^{\prime}=\theta \circ N$ and $\Sigma \theta^{\prime}=\Sigma \theta \circ \Sigma N=\ell \circ \Sigma N$.

Recalling the map $\pi_{j}$ in Definition 27 the $j$ th projected global James-Hopf invariant of $g^{\prime}$ is

$$
\gamma_{[j]}^{*}\left(g^{\prime}\right): \Sigma U \stackrel{\Sigma\left(g^{\prime}\right)^{\natural}}{\longrightarrow} \Sigma \Omega \Sigma V \stackrel{\cong h}{\rightarrow} \Sigma \bigvee_{k=1}^{\infty} V^{\wedge k} \stackrel{\pi_{j}}{\rightarrow} \Sigma S^{b_{j}} \wedge \bigvee_{l=0}^{\infty} V^{\wedge l},
$$

whose suspension is the linear map $\left(\Sigma \pi_{j}\right) \circ \ell \circ(\Sigma N)$. Since $n-a_{i}-3 \geq 1$ and since a linear map desuspends as many times as the connectivity of the spaces, we have that $\Sigma^{n-a_{i}-3} \gamma_{[j]}^{*}\left(g^{\prime}\right)$ desuspends $\left(b_{j}+1\right)$ times. Therefore by Corollary 28 the homotopy cofibre $X^{\prime}$ of $g^{\prime}$ h-embeds in $S^{n+1}$, and it is clear that $X^{\prime}$ has the rational homotopy type of $X$.

Example 44. Consider $X=\mathbb{H} P(2)=S^{4} \cup_{\nu} e^{8}$, where $\nu \in \pi_{7}\left(S^{4}\right)$ is the Hopf map. It is well known that $X$ does not h-embed in $S^{12}$, because the sphere of origin of $\{\nu\}$ is $S^{4}$ ([27], [17]). On the other hand, Theorem 7 asserts that some finite CWcomplex $X^{\prime}$ of the rational homotopy type of $X$ does h-embed in $S^{11}$. Indeed, $X^{\prime}=S^{4} \cup_{\left[S^{4}, S^{4}\right]} e^{8} \stackrel{h}{\subset} S^{11}$ by the Connolly-Williams criterion, since $\Sigma\left(\left[S^{4}, S^{4}\right]\right)=0$ . But $X^{\prime} \simeq_{\mathbb{Q}} X$ because $\left[S^{4}, S^{4}\right]=2 \nu$.

We now give some evidence for Conjecture 42 To get an h-embedding of $X$ in a sphere $S^{n+1}$ it is enough to construct a homotopy push-out like diagram (18) with $P$ a Poincaré duality space. By the techniques of [23] and [24] we can construct a homotopy push-out of rational spaces

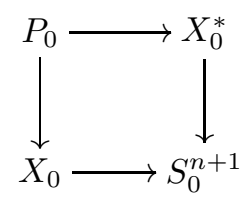

where $X_{0}$ is the rationalization of $X, S_{0}^{n+1}$ is the rationalization of the sphere, $n=\operatorname{dim} X+2$, and $P_{0}$ is a rational Poincaré duality space. We would now have to realize this homotopy push-out in "integral" homotopy theory. At least $P_{0}$ is realizable as a Poincaré duality space over $\mathbb{Z}$ by a result of [37] (this result asserts that a rational Poincaré duality space with zero-signature is realized by a closed manifold.) 


\section{Applichtion to The LS-CATEGORY AND CONE-LENGTH}

We come now to the construction of a closed manifold whose category and conelength are not affected by deleting one point, and to the proof of Proposition [13 of the Introduction. Such a manifold will be obtained as the trivial homotopy boundary of a two-cell $X=S^{p} \cup_{f} e^{q}$ such that cat $X=2$ and the adjoint map $f^{\natural}$ is stably trivial. For such a map $f$ it follows from Corollary [31 that the $n$ dimensional trivial homotopy boundary of $X$ (with $n$ sufficiently large) can be described as follows:

$$
\mathcal{B}^{n}(X)=S^{p} \cup_{f} e^{q} \vee S^{n-q} \vee S^{n-p} \cup_{\Psi} e^{n}
$$

From the computations done in [18] such a map $f$ exists. Indeed we can take for $f$ a representative of an element of order 3 in $\pi_{9}\left(S^{2}\right)$, and the space $X=S^{2} \cup_{f} e^{10}$ is then the one considered by Iwase to disprove the Ganea conjecture. It follows from the next proposition that the LS-category and cone-length of the manifold $\mathcal{B}^{n}(X)$ are not affected by the attachment of the top cell (for $n \geq 21$ ). This proves Proposition 13.

Proposition 45. Let $f: S^{q-1} \rightarrow S^{p}$ be a map with $q \geq p+2$ and let $n \geq 2 q+1$ be an integer. Denote by $X=S^{p} \cup_{f} e^{q}$ the homotopy cofibre of $f$ and suppose that cat $X=2$. If $\Sigma^{n-p+q}\left(\Sigma f^{\natural}\right)$ is homotopically trivial, then the following statements hold:

(i) $\operatorname{cat}\left(\mathcal{B}^{n}(X)\right)=\mathrm{Cl}\left(\mathcal{B}^{n}(X)\right)=\operatorname{cat} X=2$;

(ii) $\mathrm{Cl}\left(\mathcal{B}^{n}(X)\right)=\mathrm{Cl}\left(\mathcal{B}^{n}(X) \backslash\{*\}\right)$;

(iii) $\operatorname{cat}\left(\mathcal{B}^{n}(X)\right)=\operatorname{cat}\left(\mathcal{B}^{n}(X) \backslash\{*\}\right)$

Proof. Statements (ii) and (iii) are consequences of the first one and of the inequalities cat $X \leq \operatorname{cat} \mathcal{B}(X \backslash\{*\}) \leq \operatorname{cat} \mathcal{B}(X)$ and $\mathrm{Cl} X \leq \mathrm{Cl} \mathcal{B}(X \backslash\{*\}) \leq \mathrm{Cl} \mathcal{B}(X)$. We then just have to show that $\mathrm{Cl}(\mathcal{B}(X)) \leq 2$.

Observe first that if $A \rightarrow \Sigma Y \stackrel{\varrho}{\longrightarrow} Z$ is a cofibration sequence and $g: B \rightarrow \Sigma Y$ is a map, then there exists a cofibration sequence $A \vee B \rightarrow \Sigma Y \rightarrow Z \cup_{\varrho g} C B$. Therefore $\mathrm{Cl}\left(Z \cup_{\varrho g} C B\right) \leq 2$.

In order to prove the inequality $\mathrm{Cl}(\mathcal{B}(X)) \leq 2$, recall that $\Sigma \Omega S^{p} \simeq S^{p} \vee$ $\left(\Sigma \Omega S^{p} / S^{p}\right)$ and consider the cofibration sequence

$$
\begin{aligned}
\Sigma \Omega S^{p} / S^{p} & \longrightarrow \Sigma \Omega S^{p} \cup_{\Sigma f} e^{q} \vee S^{n-q} \vee S^{n-p} \\
& \stackrel{\mathrm{ev} \vee i d \vee i d}{\longrightarrow} S^{p} \cup_{f} e^{q} \vee S^{n-q} \vee S^{n-p},
\end{aligned}
$$

where $\overline{\mathrm{ev}}: \Sigma \Omega S^{p} \cup_{\Sigma f} e^{q} \rightarrow S^{p} \cup_{f} e^{q}$ is the extension to the homotopy cofibres of the evaluation map ev: $\Sigma \Omega S^{p} \rightarrow S^{p}$. Set $W=S^{n-q} \vee S^{n-p}$. We will show that the top cell attaching map $\Psi: S^{n-1} \rightarrow X \vee W=S^{p} \cup_{f} e^{q} \vee S^{n-q} \vee S^{n-p}$ factors through $\rho:=\overline{\mathrm{ev}} \vee W$. By the observation above this will prove that $\mathrm{Cl}(\mathcal{B}(X)) \leq 2$, because $\Sigma \Omega S^{p} \cup_{\Sigma f^{\natural}} e^{q} \vee S^{n-q} \vee S^{n-p}$ is a suspension. 
Consider the projections $p: S^{p} \cup_{f} e^{q} \vee W \rightarrow S^{p} \cup_{f} e^{q}$ and $p^{\prime}: \Sigma \Omega S^{p} \cup_{\Sigma f} e^{q} \vee W \rightarrow$ $\Sigma \Omega S^{p} \cup_{\Sigma f^{\natural}} e^{q}$ and the map between fibration sequences

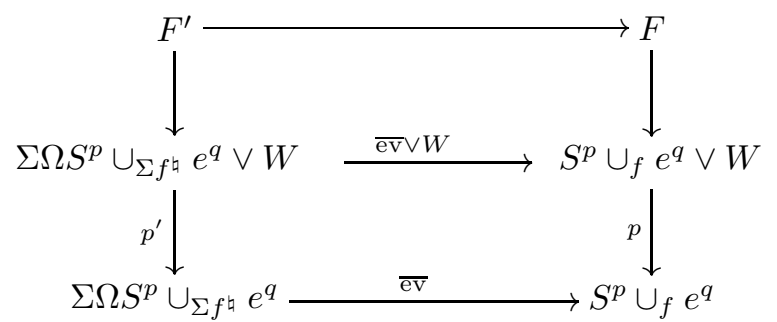

By [34, Lemmas 5.9 and 5.11, $F^{\prime} \simeq \Omega\left(\Sigma \Omega S^{p} \cup_{\Sigma f} e^{n}\right) \wedge W \vee W, F \simeq\left(\Omega\left(S^{p} \cup_{f}\right.\right.$ $\left.\left.e^{q}\right)\right) \wedge W \vee W$, and the map $F^{\prime} \rightarrow F$ is equivalent to the $\operatorname{map}(\Omega \overline{\mathrm{ev}}) \wedge W \vee W$. Since the composite $p \Psi$ is homotopically trivial, the map $\Psi$ lifts into $F$. Applying the next lemma, we see that

$$
(\Omega \overline{\mathrm{ev}})_{\#}: \pi_{q-1}\left(\Omega\left(\Sigma \Omega S^{p} \cup_{\Sigma f^{\natural}} e^{q}\right)\right) \rightarrow \pi_{q-1}\left(\Omega S^{p} \cup_{f} e^{q}\right)
$$

is surjective. Therefore $\pi_{n-1}\left(F^{\prime}\right) \rightarrow \pi_{n-1}(F)$ is also surjective and $\Psi$ lifts through $\overline{\mathrm{ev}} \vee W$. This completes the proof.

Lemma 46. There exists a map $\phi$ such that the following composite is a $(q-1)$ equivalence:

$$
\hat{\phi}:\left(\Omega S^{p}\right) \cup_{f \natural} e^{q-1} \stackrel{\phi}{\longrightarrow} \Omega\left(\Sigma \Omega S^{p} \cup_{\Sigma f^{\natural}} e^{q}\right) \stackrel{\Omega \overline{\mathrm{ev}}}{\longrightarrow} \Omega\left(S^{p} \cup_{f} e^{q}\right) .
$$

Thus $(\Omega \overline{\mathrm{ev}})_{\#}: \pi_{i}\left(\Omega\left(\Sigma \Omega S^{p} \cup_{\Sigma f^{\natural}} e^{q}\right)\right) \rightarrow \pi_{i}\left(\Omega\left(S^{p} \cup_{f} e^{q}\right)\right)$ is surjective in degrees $i \leq q-1$.

Proof. Consider the following diagram of cofibration sequences and maps between them:

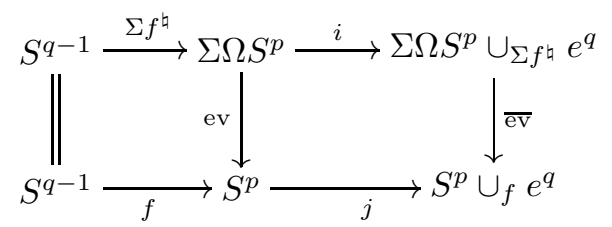

After looping, the middle vertical map has a canonical section given by the unit of adjunction. By the naturality of the adjunction we get a commutative diagram

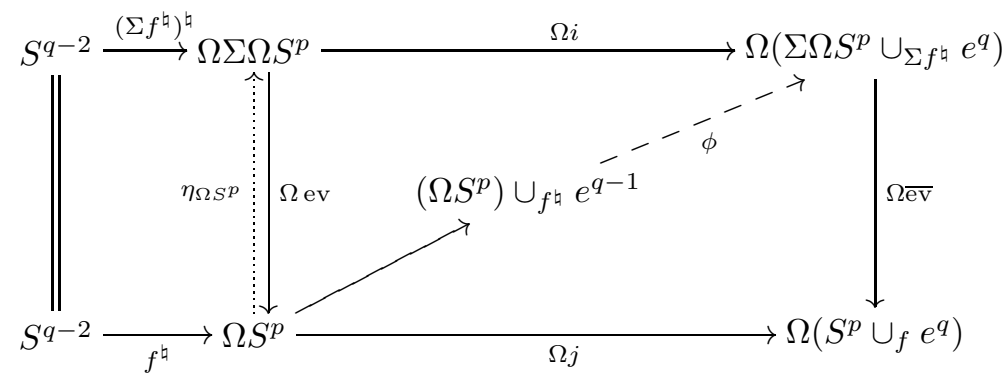

where the map $\phi$ is induced on the cofibre of $f^{\natural}$ by the map $\Omega i \circ \eta_{\Omega S^{p}}$.

An elementary computation with Adams-Hilton models shows that the composite $\hat{\phi}:=\Omega \overline{\mathrm{ev}} \circ \phi$ induces an isomorphism (resp. an epimorphism) in homology in 
degrees $<q-1$ (resp. in degree $q-1$ ). By the simple-connectivity of the spaces this implies that $\hat{\phi}$ is a $(q-1)$-equivalence. The surjectivity of $\pi_{\leq q-1}(\Omega \overline{\mathrm{ev}})$ is an immediate consequence.

\section{REFERENCES}

[1] H. Ando, On the generalized Whitehead products and the generalized Hopf invariant of a composition element. Tôhoku Math. J. (2) 20 (1968), 516-553 MR 39:2164

[2] M. Arkowitz, The generalized Whitehead product, Pacific J. Math., 12 (1962), 7-23 MR 27:5262

[3] H.J. Baues, Whitehead Produkte und Hindernisse in dem Produkt von Abbilkdungskegeln. Archiv der Math. (Basel) 25 (1974), 184-197 MR 49:11509

[4] H.J. Baues, Relationen für primäre Homotopieoperationen und eine verallgemeinerte EHP Sequenz, Ann. Sci. Ecole Normale Sup. 4, 8 (1975), 509-533 MR 53:1586

[5] H.J. Baues, Rationale Homotopietypen, Manuscripta Math. 20 (1977), no. 2, 119-131 MR 56:1297

[6] H.J. Baues, Obstruction theory, Lecture Notes in Mathematics, vol. 628, Springer-Verlag, 1977 MR 57:7600

[7] H.J. Baues, Commutator Calculus and groups of homotopy classes, London Math. Soc. Lecture Notes Series, vol. 50, Cambridge University Press, 1981 MR 83b:55012

[8] W. Browder, Embedding 1-connected manifolds, Bull. Amer. Math. Soc. 72 (1966), 225-231 MR 32:6467

[9] W. Browder, Surgery on simply-connected manifolds. Ergebnisse der Mathematik und ihrer Grenzgebiete, Band 65. Springer-Verlag, 1972 MR 50:11272

[10] F. Connolly, B. Williams, Embedding up to homotopy type and geometric suspensions of manifolds, Quarterly J. Math. Oxford (2), 29 (1978), 385-401 MR 83a:57022

[11] G. Cooke, Embedding certain complexes up to homotopy type in Euclidean space, Annals of Math. (2), 90 (1969), 144-156 MR 39:3486

[12] G. Cooke, Thickenings of $C W$ complexes of the form $S^{m} \cup_{\alpha} e^{n}$, Trans. Amer. Math. Soc. 247 (1979), 177-210 MR 81c:55016

[13] O. Cornea, Cone-Length and Lusternik-Schnirelmann category, Topology 33 (1994), 95-111 MR 95d:55008

[14] O. Cornea, Y. Félix, J.-M. Lemaire, Rational LS-category and cone-length of Poincaré duality complexes, Topology 37 (1998), 743-748 MR 99a:55022

[15] T. Ganea A generalization of the homology and homotopy suspension, Comment. Math. Helveticii 39 (1965), 295-322 MR 31:4033

[16] N. Habegger, Embedding up to homotopy type-the first obstruction, Topology Appl. 17 (1984), no. 2, 131-143 MR 85e:57022

[17] P. Hilton, E. Spanier, On the embeddability of certain complexes in the euclidean space, Proc. Amer. Math. Soc. 11 (1960), 523-526 MR 23:A2211

[18] N. Iwase, Ganea's conjecture on Lusternik-Schnirelmann category, Bull. London Math. Soc. 30 (1998), no. 6, 623-634 MR 99j:55003

[19] N. Iwase, Lusternik-Schnirelmann category of a sphere bundle over a sphere, preprint 2001

[20] I.M. James, Reduced product spaces, Annals of Math. (2), 62 (1955), 170-197 MR 17:396b

[21] I.M. James, Note on cup product, Proc. Amer. Math. Soc. 8 (1957), 374-383 MR 19:974a

[22] J. Klein, On the homotopy embeddability of complexes in euclidean space. I. The weak thickening theorem, Math. Z. 213 (1993), no. 1, 145-161 MR 95e:57042

[23] P. Lambrechts, Cochain models of thickenings and applications to rational LS-category, Manuscripta Math. 103 (2000), no. 2, 143-160 MR 2001m:55009

[24] P. Lambrechts and L. Vandembroucq, Modeles de Quillen des bords homotopiques, http://gauss.math.ucl.ac.be/ ${ }^{2}$ ambrech/publications.html, preprint 1999

[25] M. Mahowald, A new infinite family in ${ }_{2} \pi_{*}^{S}$, Topology 16 (1977), no. 3, 249-256 MR 56:3838

[26] M. Mahowald, Private communication

[27] F.P. Peterson, Some non-embedding problems, Bol. Soc. Mat. Mexicana (2) 2 (1957), 9-15 MR 19:440d

[28] F.P. Peterson and N. Stein, The dual of a secondary operation, Illinois J. Math. 4 (1960), 397-404 MR 27:1948 
[29] J.J. Rivadeneyra-Perez, On cat $(X \backslash p)$, International J. Math. and Math. Sci. 15 (1992), 465-468 MR 93g:55005a

[30] D. Ravenel, Private communication

[31] C.P. Rourke, B.J. Sanderson, Introduction to Piecewise-Linear Topology, Ergebnisse der Mathematik und ihrer Grenzgebiete, Band 69, Springer Verlag, 1972 MR 50:3236

[32] A. Shapiro, Obstructions to the embedding of a complex in a euclidian space. I. The first obstruction, Ann. of Math. (2) 66 (1957), 256-269 MR 19:671a

[33] E. Spanier, Function spaces and duality, Ann. of Math. (2) 70 (1959), 338-378 MR 21:6584

[34] D. Stanley, Spaces with Lusternik-Schnirelmann category $n$ and cone-length $n+1$, Topology 39 (2000), no. 5, 985-1019 MR 2001e:55004

[35] N. Steenrod and D. Epstein, Cohomology operations, Annals of Math. studies, Vol. 50, Princeton University Press, 1962 MR 26:3056

[36] J. Stallings, The embedding of homotopy types into manifolds, mimeo notes, Princeton University, 1966

[37] D. Sullivan, Infinitesimal computations in topology, Inst. Hautes Études Sci. Publ. Math. No. 47, (1977), 269-331 MR 58:31119

[38] R. Switzer, Algebraic topology - homotopy and homology, Die Grundlehren der mathematischen Wissenschaften, Band 212. Springer-Verlag, New York-Heidelberg, 1975. xii+526 pp. MR 52:6695

[39] R. Thom, Espaces fibrés en sphères et carrés de Steenrod, Ann. Sci. Ecole Normale Sup. 69 (1952), pp. 109-182 MR 14:1004c

[40] C.T.C. Wall, Classification problems in differential topology IV: thickenings, Topology 5 (1966), pp. 73-94 MR 33:734

[41] G. Whitehead, Elements of Homotopy Theory, Graduate Texts in Math., vol. 61, SpringerVerlag, 1978 MR 80b:55001

Laboratoire de GÉométrie-Algèbre “LaboGA” DE L'Université D'Artois

Current address: Institut Mathématique, 2 Chemin du Cyclotron, B-1348 Louvain-la-Neuve, Belgium

E-mail address: lambrechts@math.ucl.ac.be

Department of Mathematical Sciences, University of Alberta, Edmonton, Alberta, Canada T6G 2G1

E-mail address: stanley@math.ualberta.ca

Universidade do Minho, CMat, Departamento de Matemática, 4710 Braga, Portugal

E-mail address: lucile@math.uminho.pt 